\title{
La Producción de Electricidad con Energía Solar Fotovoltaica en España en el Siglo XXI
}

\author{
The Production of Electricity with Photovoltaic Solar Energy in Spain \\ in the 21st Century
}

\author{
Cayetano Espejo-Marín \\ cespejo@um.es O https://orcid.org/0000-0001-6614-1365 \\ Universidad de Murcia. Departamento de Geografía. Campus de La Merced. 30001 Murcia \\ Ana Eulalia Aparicio-Guerrero \\ Ana.Aparicio@uclm.es@ https://orcid.org/0000-0002-1011-7566 \\ Universidad de Castilla-La Mancha. Departamento de Geografía y Ordenación del Territorio. Facultad de Ciencias de la \\ Educación y Humanidades. Avda. de los Alfares, 44. 16071 Cuenca
}

\section{INFO ARTÍCULO}

Recibido: 01/04/2019

Revisado: 16/09/2019

Aceptado: 03/12/2019

\section{PALABRAS CLAVE}

Producción Eléctrica

Energía Solar Fotovoltaica

Política Energética

España

\begin{abstract}
RESUMEN
La implantación y desarrollo de centrales de energía solar fotovoltaica en España ha sido posible por las políticas de fomento de las energías renovables de la Unión Europea, así como por la disponibilidad de abundante radiación solar. Los Planes de Energías Renovables y las medidas de apoyo a esta tecnología por parte del Gobierno de España contribuyeron a su espectacular crecimiento a mediados de la primera década de este siglo. Con posterioridad, una nueva regulación llevó a la contención y casi paralización del sector hasta fechas recientes. En la actualidad se vive una etapa de desarrollo consecuencia de la nueva apuesta por las energías renovables para cumplir con las directrices comunitarias y por el desarrollo del autoconsumo. Este trabajo tiene como objetivo realizar un análisis sobre el proceso de implantación de la energía solar fotovoltaica en España, su desarrollo y su situación actual. El método utilizado se ha centrado en el análisis de las consecuencias de las decisiones de los Gobiernos de España en relación con este sector energético, en lo referente a la evolución de la potencia instalada y a su distribución territorial. Los resultados confirman la clara relación entre la política de incentivos y su expansión en la etapa inicial, la política de contención y estabilización durante una década, y la situación actual de nueva recuperación, que viene motivada por el progreso tecnológico y por el desarrollo del autoconsumo, favorecidos en ambos casos por la enorme reducción del coste de las instalaciones.
\end{abstract}

\section{KEYWORDS}

Electrical Production Photovoltaic Solar Energy, Energy Policy Spain.

\begin{abstract}
Subsequently, a new Government regulation led to the containment and almost stagnation of the sector until recent dates. At present, a stage of development is taking place as a result of the new commitment to renewable energies to comply with the EU guidelines and the development of self-consumption.

The objective of this work is to carry out an analysis of the process of implementation of photovoltaic solar energy in Spain, its development and its current situation.

The method used has focused on the analysis of the consequences of the decisions of the Governments of Spain in relation to this energy sector, with regard to the evolution of the installed power and its territorial distribution. The results confirm the clear relationship between the policy of incentives and their expansion in the initial stage, the policy of containment and stabilization for a decade and the current situation of new recovery, which is motivated by technological progress and the development of self-consumption, favored in both cases by the enormous reduction in the cost of the facilities.
\end{abstract}




\section{INTRODUCCIÓN}

Desde finales del siglo XX, para cumplir con las directrices de la Unión Europea en lo referente al consumo de energías renovables, España ha apostado claramente por estas tecnologías para la producción de electricidad. Dentro de ellas predominan, por potencia instalada y distribución territorial, la eólica, la solar fotovoltaica y la solar termoeléctrica. Su desarrollo ha sido posible gracias a la existencia de un marco legal y económico muy favorable para su implantación (Galdós, R. y Madrid, J. 2009; Espejo, C. y García, R., 2010 y 2012; Espejo, C., 2012; Prados, M.J. et al., 2012; Frolova, M. et al., 2014).

En el caso de la solar fotovoltaica, además de la abundante radiación con la que cuenta todo el territorio español, han jugado un papel fundamental las entidades financiares porque han aportado los recursos económicos necesarios para que decenas de miles de inversores pudieran invertir en este tipo de instalaciones, a través de un sistema singular de agrupación de las mismas denominado huerto solar, que constituye la base de la estructura productiva de la energía solar fotovoltaica en España. España ha sido pionera a nivel mundial en la implantación de la energía solar fotovoltaica (Espejo, C., 2004; Mir, P., 2012; Mérida, M. et al., 2015), experimentó un crecimiento espectacular durante los años 2007 y 2008, posteriormente una fase de estancamiento que ha durado una década, y en la actualidad atraviesa otro momento de importante impulso.

Teniendo en cuenta estas premisas y la metodología empleada, la presente investigación tiene como objetivos realizar un análisis sobre el proceso de implantación de la energía solar fotovoltaica en España, su desarrollo y situación actual.

\section{METODOLOGÍA}

La metodología se basa en la consecución de las siguientes fases: a) Analizar la producción de electricidad en España, b) Recopilar el marco legal y económico de la producción de electricidad de origen solar fotovoltaico; c) Estudiar la producción de la energía solar fotovoltaica en España; d) Exponer las medidas recientes para el apoyo a esta tecnología energética; y e) Describir las causas principales de la expansión de la energía solar fotovoltaica a nivel mundial.

En este sentido, y de acuerdo con Salkind y Rainwater (2003), la metodología seguida se encuadra en la categoría de no experimental, subcategorías descriptiva, histórica y correlacional, examinando las relaciones entre variables o datos aportados por diferentes organismos, empresas y asociaciones: Instituto para la Diversificación y Ahorro de la Energía, Red Eléctrica de España, Comisión Nacional de los Mercados y la Competencia, Asociación de Productores de Energías Renovables, etc.

Para alcanzar el objetivo propuesto se ha realizado una revisión de las referencias bibliográficas básicas necesarias, generales sobre energías renovables y específicas sobre la energía solar fotovoltaica en España. Fundamental ha sido la revisión de una serie de documentos clave, entre los que destacan: los Planes de Energías Renovables, las leyes y decretos que emanan de los mismos, informes de la Comisión Nacional de los Mercados y la Competencia, y otros documentos estadísticos.

Toda esta información ha permitido la elaboración de 18 tablas en las que se exponen de forma sintetizada los datos más relevantes de los aspectos analizados en cada apartado.

El artículo ha quedado estructurado en las siguientes partes: a) una presentación de la estructura del parque generador de electricidad en España y del papel que tienen las energías renovables, b) una recopilación de todo el marco jurídico-económico que ha afectado a la energía solar fotovoltaica desde finales del siglo pasado; c) un análisis de la evolución de la potencia instalada y de su relevancia en la Unión Europea y en el Planeta, así como de la producción de electricidad a lo largo de este siglo, d) una exposición de la distribución territorial por Comunidades Autónomas de la potencia y de la producción de electricidad, e)una relación de medidas recientes de apoyo a la energía solar fotovoltaica, y f) la evolución de los precios de los componentes de un sistema fotovoltaico. 


\section{RESULTADOS}

\subsection{La producción de electricidad en España}

Dentro del consumo de energía final en España, la electricidad supone casi una cuarta parte del total (Tabla 1), y según el consumo de energía primaria las renovables van teniendo una relevancia cada vez mayor, aunque oscila cada año en función de la producción de energía hidráulica. La energía solar fotovoltaica ha aportado en el año 2017 el 0,6\% del total y el 4,7 \% de las renovables (Tabla 2).

Tabla 1. Consumo de energía final en España. Kilotoneladas equivalentes de petróleo (ktep). Años 2016 y 2017.

\begin{tabular}{|l|c|c|c|c|}
\hline \multirow{2}{*}{\multicolumn{1}{|c|}{ Tipo de energía }} & \multicolumn{2}{c|}{2016} & \multicolumn{2}{c|}{2017} \\
\cline { 2 - 5 } & Ktep & $\%$ & Ktep & 51,31 \\
\hline Productos petrolíferos & 42.197 & 50,99 & 43.005 & 24,06 \\
\hline Electricidad & 19.993 & 24,16 & 20.169 & 15,58 \\
\hline Gas & 13.445 & 16,25 & 13.059 & 6,86 \\
\hline Energías renovables & 5.523 & 6,67 & 5.748 & 2,19 \\
\hline Carbón & 1.598 & 1,93 & 1.838 & $\mathbf{8 3 . 8 1 9}$ \\
\hline Total & $\mathbf{8 2 . 7 5 6}$ & $\mathbf{1 0 0 , 0 0}$ & $\mathbf{1 0 0 , 0 0}$ \\
\hline
\end{tabular}

Fuente: Instituto para la Diversificación y Ahorro de la Energía. Informe Estadístico Energías Renovables.

Tabla 2. Consumo de energía primaria en España. Kilotoneladas equivalentes de petróleo (ktep). Años 2016 y 2017.

\begin{tabular}{|c|c|c|c|c|}
\hline \multirow{2}{*}{ Tipo de energía } & \multicolumn{2}{|c|}{2016} & \multicolumn{2}{|c|}{2017} \\
\hline & Ktep & $\%$ & Ktep & $\%$ \\
\hline Petróleo & 55.506 & 44,4 & 57.969 & 44,3 \\
\hline Gas natural & 25.040 & 20,0 & 27.274 & 20,9 \\
\hline Nuclear & 15.273 & 12,2 & 15.119 & 11,6 \\
\hline Carbón & 10.901 & 8,7 & 12.821 & 9,8 \\
\hline Energías Renovables: & 17.462 & 14,0 & 16.509 & 12,6 \\
\hline - Biomasa & 5.327 & 4,3 & 5.499 & 4,2 \\
\hline - Eólica & 4.204 & 3,4 & 4.224 & 3,2 \\
\hline - Hidráulica & 3.130 & 2,5 & 1.615 & 1,2 \\
\hline — Solar termoeléctrica & 2.190 & 1,8 & 2.310 & 1,8 \\
\hline - Biocarburantes & 1.125 & 0,9 & 1.290 & 1,0 \\
\hline - Solar fotovoltaica & 694 & 0,6 & 729 & 0,6 \\
\hline - Solar térmica & 293 & 0,2 & 309 & 0,2 \\
\hline - Biogás & 245 & 0,2 & 254 & 0,2 \\
\hline — Res. sólidos urbanos & 235 & 0,2 & 260 & 0,2 \\
\hline - Geotérmica & 19 & 0,0 & 19 & 0,0 \\
\hline Saldo eléctrico & 659 & 0,5 & 788 & 0,6 \\
\hline Total & 125.076 & 100,0 & 130.739 & 100,0 \\
\hline
\end{tabular}

Fuente: Instituto para la Diversificación y Ahorro de la Energía. Informe Estadístico Energías Renovables. 
La estructura productiva de energía eléctrica en España se caracteriza por la diversidad de tecnologías empleadas, el predominio de las tradicionales y por tanto no renovables, y una presencia cada vez más relevantes de las energías renovables. Al final del año 2018 España contaba con una potencia instalada superior a los 104.000 megavatios (MW), de los cuales el 95\% corresponden al Sistema Peninsular y el 5\% a los Sistemas Extrapeninsulares, que incluyen Baleares, Canarias, Ceuta y Melilla (Tabla 3). La distribución de la capacidad productiva presenta situaciones distintas en cada caso. En la Península, las centrales de ciclo combinado, que consumen gas natural, acumulan una cuarta parte de la capacidad (24,9\%); les siguen los parques eólicos con el 23,4\% y la hidráulica, que aporta el 20,6\%. Las otras tecnologías con un papel relevante son las centrales de carbón (9,7\%), las centrales nucleares (7,2\%), las plantas de cogeneración (5,8\%), las instalaciones fotovoltaicas $(4,5 \%)$ y las centrales solares termoeléctricas (2,3\%). En el Sistema Extrapeninsular las centrales de fuel/gas acumulan el $45,7 \%$ de la potencia instalada. Abastecen en su totalidad a Ceuta, Melilla y las islas de tamaño más reducido; les siguen en importancia las plantas de ciclo combinado (31,6\%), la central de carbón de importación de la isla de Mallorca en Baleares (8,6\%), las instalaciones eólicas de Canarias (7,6\%) y la solar fotovoltaica (4,5\%); el resto centrales suman únicamente un $2 \%$, y en ellas se incluye la central hidroeólica de la isla de El Hierro en Canarias.

Tabla 3. Potencia de energía eléctrica instalada en España según tecnologías. MW. 31-12-2018.

\begin{tabular}{|c|c|c|c|c|c|c|}
\hline & \multicolumn{2}{|c|}{ Sistema peninsular } & \multicolumn{2}{|c|}{ Sistemas no peninsulares* } & \multicolumn{2}{|c|}{ Total } \\
\hline & MW & $\%$ & MW & $\%$ & MW & $\%$ \\
\hline Hidráulica & 20.376 & 20,66 & 2 & 0,04 & 20.378 & 19,58 \\
\hline Nuclear & 7.117 & 7,21 & 0 & 0,00 & 7.117 & 6,84 \\
\hline Carbón & 9.562 & 9,69 & 468 & 8,59 & 10.030 & 9,64 \\
\hline Fuel/gas & 0 & 0,00 & 2.490 & 45,70 & 2.490 & 2,39 \\
\hline Ciclo combinado & 24.562 & 24,90 & 1.722 & 31,60 & 26.284 & 25,25 \\
\hline Hidroeólica & 0 & 0,00 & 11 & 0,20 & 11 & 0,01 \\
\hline Eólica & 23.091 & 23,41 & 416 & 7,63 & 23.507 & 22,58 \\
\hline Solar fotovoltaica & 4.465 & 4,53 & 248 & 4,55 & 4.713 & 4,53 \\
\hline Solar térmica & 2.304 & 2,34 & 0 & 0,00 & 2.304 & 2,21 \\
\hline Otras renovables** & 859 & 0,87 & 6 & 0,11 & 865 & 0,83 \\
\hline Cogeneración & 5.730 & 5,81 & 10 & 0,18 & 5.740 & 5,51 \\
\hline Residuos no renovables & 452 & 0,46 & 38 & 0,70 & 490 & 0,47 \\
\hline Residuos renovables & 123 & 0,12 & 38 & 0,70 & 161 & 0,15 \\
\hline Total & 98.643 & 100,00 & 5.449 & 100,00 & 104.092 & 100,00 \\
\hline
\end{tabular}

* Canarias, Baleares, Ceuta y Melilla.

** Incluye biogás, biomasa, hidráulica marina y geotérmica.

Fuente: Red Eléctrica de España. El Sistema Eléctrico Español. 2018.

La producción de electricidad está condicionada por la demanda, la estructura del parque generador y, debido al gran peso de la hidráulica y de la eólica, por la disponibilidad de agua y la existencia de vientos en calidad y cantidad suficiente para su aprovechamiento (Espejo, C. et al., 2010, 2012). En años con escasas precipitaciones se incrementa el papel en la producción de las centrales de ciclo combinado y de carbón, preferentemente las que consumen carbón de importación. Por consiguiente, la diversificación del parque generador eléctrico español lleva a que la demanda se cubra con una variedad de fuentes (Tabla 4). 
Tabla 4. Producción de energía eléctrica en España según tecnologías. Gigavatios/hora (GWh). 31-12-2018.

\begin{tabular}{|c|c|c|c|c|c|c|}
\hline & \multicolumn{2}{|c|}{ Sistema peninsular } & \multicolumn{2}{|c|}{ Sistemas no peninsulares* } & \multicolumn{2}{|c|}{ Total } \\
\hline & GWh & $\%$ & GWh & $\%$ & GWh & $\%$ \\
\hline Hidráulica & 36.112 & 14,63 & 3 & 0,02 & 36.115 & 13,84 \\
\hline Nuclear & 53.198 & 21,55 & 0 & 0,00 & 53.198 & 20,38 \\
\hline Carbón & 34.882 & 14,13 & 2.392 & 16,99 & 37.274 & 14,28 \\
\hline Fuel/gas & 0 & 0,00 & 6.683 & 47,46 & 6.683 & 2,56 \\
\hline Ciclo combinado & 26.403 & 10,69 & 3.642 & 25,86 & 30.045 & 11,51 \\
\hline Hidroeólica & 0 & 0,00 & 24 & 0,17 & 24 & 0,01 \\
\hline Eólica & 48.946 & 19,82 & 625 & 4,44 & 49.571 & 18,99 \\
\hline Solar fotovoltaica & 7.374 & 2,99 & 385 & 2,73 & 7.759 & 2,97 \\
\hline Solar térmica & 4.424 & 1,79 & 0 & 0,00 & 4.424 & 1,70 \\
\hline Otras renovables** & 3.547 & 1,44 & 10 & 0,07 & 3.557 & 1,36 \\
\hline Cogeneración & 28.981 & 11,74 & 35 & 0,25 & 29.016 & 11,12 \\
\hline Residuos no renovables & 2.294 & 0,93 & 141 & 1,00 & 2.435 & 0,93 \\
\hline Residuos renovables & 733 & 0,30 & 141 & 1,00 & 874 & 0,33 \\
\hline Total & 246.894 & 100,00 & 14.081 & 100,00 & 260.975 & 100,00 \\
\hline
\end{tabular}

* Canarias, Baleares, Ceuta y Melilla.

** Incluye biogás, biomasa, hidráulica marina y geotérmica.

Fuente: Red Eléctrica de España. El Sistema Eléctrico Español. 2018.

Los efectos de la crisis económica que ha padecido España desde 2007 a 2014 se han reflejado en muchas variables. Una de ellas ha sido la evolución del consumo de electricidad en la España Peninsular (Tabla 5). En el sistema no peninsular, Ceuta y Melilla no han tenido modificaciones apreciables. En cambio, en los espacios insulares el crecimiento del turismo como motor de sus actividades económicas ha provocado un aumento en el consumo eléctrico, que se ha atendido en cada caso de un modo distinto. En Baleares, las islas de Mallorca, Menorca e Ibiza están interconectadas, y para garantizar su abastecimiento se construyó un cable de conexión con la Península entre Sagunto-Valencia- y Calviá-Mallorca (Red Eléctrica de España, 2011). En Canarias las centrales de ciclo combinado han sustituido los antiguos grupos de fuel por plantas de mayor capacidad y eficiencia energética, así como menos contaminantes.

El segundo hecho que singulariza el sistema eléctrico español es la abundante presencia de instalaciones de producción eléctrica con energías renovables. Su aportación a la producción eléctrica nacional se ha duplicado en una década (Tabla 6). Aunque en años de sequía y su repercusión en la producción hidroeléctrica, como en 2017, se reduce considerablemente su papel.

El desarrollo de las energías renovables en España no se ha dado de igual modo en las tecnologías empleadas y en el momento de su implantación (Tabla 7). Cada tecnología ha llevado una evolución y en lo que coinciden todas es en la situación de estancamiento que viven durante los últimos años, como consecuencia de las medidas de política energética adoptadas por el Gobierno de España. 
Tabla 5. Evolución de la demanda peninsular de electricidad Teravatios/ hora (TWh) y del Producto Interior Bruto en España. \%. 2007-2018.

\begin{tabular}{|l|l|l|l|}
\hline Año & TWH & Demanda & 3,8 \\
\hline 2007 & 262 & 4,3 & 1,1 \\
\hline 2008 & 265 & 1,1 & $-3,6$ \\
\hline 2009 & 253 & $-4,5$ & 0,0 \\
\hline 2010 & 261 & 3,2 & $-1,0$ \\
\hline 2011 & 256 & $-1,9$ & $-2,9$ \\
\hline 2012 & 252 & $-1,6$ & $-1,7$ \\
\hline 2013 & 246 & $-2,4$ & 1,4 \\
\hline 2014 & 244 & $-0,8$ & 3,6 \\
\hline 2015 & 248 & 1,6 & 3,2 \\
\hline 2016 & 250 & 0,8 & 3,0 \\
\hline 2017 & 253 & 1,2 & 2,6 \\
\hline
\end{tabular}

Fuente: Red Eléctrica de España. El Sistema Eléctrico Español. 2018, y Diario Expansión: https://datosmacro.expansion.com/pib/espana.

Tabla 6. Aportación de la generación eléctrica renovable y no renovable. 2007-2018.

\begin{tabular}{|c|c|c|}
\hline Año & Renovables* & No renovables** \\
\hline 2007 & 21,3 & 78,7 \\
\hline 2008 & 21,6 & 78,4 \\
\hline 2009 & 27,9 & 64,7 \\
\hline 2010 & 35,3 & 67,5 \\
\hline 2011 & 32,5 & 68,1 \\
\hline 2012 & 31,9 & 57,7 \\
\hline 2013 & 42,3 & 57,2 \\
\hline 2014 & 42,8 & 63,1 \\
\hline 2015 & 36,9 & 58,2 \\
\hline 2016 & 40,8 & 66,3 \\
\hline 2017 & 33,7 & 59,7 \\
\hline 2018 & 40,1 & 52,3 \\
\hline
\end{tabular}

* Hidráulica, eólica, solar fotovoltaica, solar térmica y otras renovables y el 50\% de los residuos sólidos urbanos.

** Nuclear, carbón, fuel/gas, ciclo combinado, cogeneración y residuos.

Fuente: Red Eléctrica de España. El Sistema Eléctrico Español. 2018. 
Tabla 7.Potencia de energía eléctrica instalada en España con tecnologías renovables. MW. 1998-2018.

\begin{tabular}{|c|c|c|c|c|c|}
\hline Año & Biomasa & Eólica & Hidráulica* & Solar Fotovoltaica & Solar Termoeléctrica \\
\hline 1998 & 81 & 886 & 1.296 & 1 & - \\
\hline 1999 & 88 & 1.686 & 1.436 & 2 & - \\
\hline 2000 & 148 & 2.296 & 1.466 & 2 & - \\
\hline 2001 & 231 & 3.508 & 1.559 & 4 & - \\
\hline 2002 & 353 & 5.066 & 1.591 & 7 & - \\
\hline 2003 & 455 & 6.324 & 1.664 & 11 & - \\
\hline 2004 & 470 & 8.532 & 1.706 & 23 & - \\
\hline 2005 & 500 & 10.095 & 1.768 & 47 & - \\
\hline 2006 & 541 & 11.897 & 1.898 & 146 & - \\
\hline 2007 & 557 & 14.536 & 1.895 & 690 & 11 \\
\hline 2008 & 587 & 16.323 & 1.979 & 3.398 & 61 \\
\hline 2009 & 670 & 18.857 & 2.015 & 3.391 & 232 \\
\hline 2010 & 709 & 19.700 & 2.026 & 3.829 & 532 \\
\hline 2011 & 736 & 21.064 & 2.031 & 4.232 & 999 \\
\hline 2012 & 810 & 22.631 & 2.032 & 4.522 & 1.950 \\
\hline 2013 & 700 & 22.994 & 2.090 & 4.645 & 2.299 \\
\hline 2014 & 739 & 23.015 & 2.093 & 4.654 & 2.299 \\
\hline 2015 & 747 & 23.031 & 2.104 & 4.666 & 2.299 \\
\hline 2016 & 747 & 23.066 & 2.106 & 4.673 & 2.299 \\
\hline 2017 & 749 & 23.094 & 2.094 & 4.678 & 2.299 \\
\hline 2018 & 750 & 23.151 & 2.094 & 4.698 & 2.299 \\
\hline
\end{tabular}

* Incluye las centrales con una potencia instalada de hasta $10 \mathrm{MW}$.

Fuente: Comisión Nacional de los Mercados y la Competencia.

Según Villarig (2013), el sector de las energías renovables en España, motor de crecimiento económico y de desarrollo territorial, fomenta el desarrollo económico de muchas zonas rurales por el carácter distribuido de la generación de energía de las diferentes tecnologías, genera grandes beneficios al conjunto de la sociedad española, y juega un papel fundamental en la lucha contra el cambio climático. Su crecimiento ha contribuido a alcanzar los objetivos medioambientales del país y a conseguir los objetivos globales energéticos. También hay que tener en cuenta su papel en la reducción de la histórica dependencia energética, que en el caso de España está muy lejos de la media de la Unión Europea (Tabla 8), al ser las únicas tecnologías de generación autóctona y limpia. 
Tabla 8. Dependencia energética de España y de la Unión Europea 2007-2017.

\begin{tabular}{|c|c|c|}
\hline Año & España & Unión Europea \\
\hline 2007 & 81,3 & 54,7 \\
\hline 2008 & 79,6 & 52,9 \\
\hline 2009 & 79,1 & 53,7 \\
\hline 2010 & 76,7 & 54,0 \\
\hline 2011 & 76,3 & 53,3 \\
\hline 2012 & 73,1 & 53,1 \\
\hline 2013 & 70,4 & 53,4 \\
\hline 2014 & 72,9 & 54,0 \\
\hline 2015 & 73,3 & 54,0 \\
\hline 2016 & 72,3 & 53,6 \\
\hline
\end{tabular}

Fuente: Asociación de Productores de Energías Renovables. Estudio del Impacto Macroeconómico de las Energías Renovables en España 2017.

Los estados europeos han utilizado diferentes instrumentos para apoyar la inversión en energías renovables: los basados en el sistema de precios, en el establecimiento de cuotas o en medidas fiscales. Las medidas apoyadas en precios o primas a las renovables subvencionan las energías renovables por encima del precio de mercado de la electricidad convencional. Los mecanismos que establecen cuotas exigen a los generadores o comercializadores de electricidad mantener un porcentaje mínimo de renovables en su cartera de generación. Y los instrumentos fiscales apoyan directamente la inversión en instalaciones renovables bien mediante subvenciones a la inversión o con desgravaciones fiscales. Los instrumentos de precios son los más utilizados en Europa y consisten en pagos fijos o una prima sobre el precio de la electricidad generado con fuentes renovables. Un esquema de este tipo ha estado vigente en Alemania, Dinamarca y España, entre otros países (Delgado, J., 2013).

\subsection{El marco legal-económico de desarrollo para la energía solar fotovoltaica}

Desde finales del siglo pasado dos Leyes del Sector Eléctrico y varios Reales Decretos han regulado en España la producción de electricidad con energías renovables y por tanto la solar fotovoltaica.

El origen del apoyo a las energías renovables tiene lugar con la aprobación de la Ley 54/1997', de 27 de noviembre, del Sector Eléctrico. Con esta Ley se establece un régimen especial para la producción de energía eléctrica cuando se realice en instalaciones cuya potencia instalada no supere los $50 \mathrm{MW}$, siempre que utilicen:

a. La cogeneración u otras formas de producción de electricidad asociadas a actividades no eléctricas siempre que supongan un alto rendimiento energético.

b. Como energía primaria alguna de las energías renovables no consumibles, biomasa o cualquier tipo de biocarburante, siempre y cuando su titular no realice actividades de producción en el régimen ordinario.

c. Como energía residuos no renovables.

1. Boletín Oficial del Estado de 28 de noviembre de 1997, $n^{\circ} 285, \mathrm{pp} .35 .097-35.126$. 
También tiene la consideración de producción en régimen especial la producción de energía eléctrica desde instalaciones de tratamiento y reducción de los residuos de los sectores agrícola, ganadero y de servicios, con una potencia instalada igual o inferior a $25 \mathrm{MW}$, cuando supongan un alto rendimiento energético.

Además, para que en el año 2010 las fuentes de energías renovables cubrieran como mínimo el 12\% de la demanda de energía total en España, se estableció la necesidad de elaborar un Plan de Fomento de las Energías Renovables, cuyos objetivos serían tenidos en cuenta en la fijación de las primas a la producción.

El Real Decreto 2818/19982, de 23 de diciembre, tiene como objeto el desarrollo reglamentario del régimen especial de la Ley 54/1997 del Sector Eléctrico. En él se establece que los titulares de las instalaciones con potencia igual o inferior a $50 \mathrm{MW}$ no tendrán la obligación de formular ofertas al mercado mayorista para dichas instalaciones, pero tendrán el derecho a vender sus excedentes o la producción de energía eléctrica a los distribuidores al precio final horario medio del mercado de producción de energía eléctrica, complementado, en su caso, por una prima o incentivo.

En su artículo 20.5 se indica que "siempre que sea posible se procurará que varias instalaciones productoras utilicen las mismas instalaciones de evacuación de la energía eléctrica, aun cuando se trate de titulares distintos. Los órganos de la Administración competente, cuando autoricen esta utilización, fijarán las condiciones que deben cumplir los titulares a fin de no desvirtuarse las medidas de energía eléctrica excedentaria de cada una de las instalaciones de producción que utilicen dichas instalaciones de evacuación". Este hecho se ha considerado como una causa de proliferación de las plantas de suelo frente a las de techo (De la Hoz et al., 2010).En efecto, los promotores impulsaron plantas de hasta 5 kilovatios pico (kWp) que se reunían en parques de mucha mayor potencia lo cual, por una parte, garantizaba obtener la máxima retribución a sus titulares y, por la otra, aprovechaban las economías de escala dado que los sistemas de conexión, los equipos de control del perímetro del recinto, los accesos a la instalación, etc. eran comunes. El sector fotovoltaico, pues, ganó experiencia en el montaje de plantas en suelo que resultaban de la yuxtaposición de los denominados huertos solares (Mir, P., 2012).

El Plan de Fomento de las Energías Renovables en España 2000-2010, aprobado por el Gobierno el 30 diciembre de 1999, articulaba una estrategia para que el crecimiento de la energía renovable pueda cubrir, en su conjunto, cuando menos, el $12 \%$ del consumo de energía primaria en el año 2010.El objetivo fijado para el sector de la energía solar fotovoltaica es de $135 \mathrm{MW}$, de los que 20 corresponden a instalaciones aisladas y 115 a instalaciones conectadas a la red (Instituto para la Diversificación y Ahorro de la Energía, 1999).

El Real Decreto 436/2004³, de 12 de marzo, tiene por objeto:

- La actualización, sistematización y refundición de las disposiciones reglamentarias dictadas en desarrollo de las normas sobre régimen jurídico de la actividad de producción de energía eléctrica en régimen especial recogidas en la Ley 54/1997 del sector eléctrico.

- El establecimiento de un régimen económico duradero para las instalaciones acogidas al régimen especial, basado en una metodología de cálculo de la retribución, objetiva y transparente, compatible con la metodología para la aprobación o modificación de la tarifa eléctrica media o de referencia, regulada por el Real Decreto 1432/2002.

- El establecimiento de sendos regímenes económicos transitorios, tanto para las instalaciones acogidas al Real Decreto 2366/1994 y al Real Decreto 2818/1998.

- La determinación de una prima complementaria para aquellas instalaciones mayores de 50 MW, de acuerdo con lo establecido en el artículo 30.5 de la Ley 54/1997 de Sector Eléctrico.

El 26 de agosto de 2005 el Gobierno de España aprueba el Plan de Energías Renovables 2005-2010, con el fin de reforzar los objetivos prioritarios de su Política Energética, aumentar la seguridad y calidad del suministro eléctrico y mejorar el respeto al medio ambiente. También se pretende dar cumplimiento a los compromisos internacionales que para España derivan del Protocolo de Kioto, así como de nuestra pertenencia a la Unión Europea, y alcanzar los objetivos del Plan Nacional de Asignación de derechos de 
emisión de gases de efecto invernadero 2008-2012.Este Plan conlleva la revisión del Plan de Fomento de las Energías Renovables en España 2000-2010, y se establece debido a que el crecimiento de algunas tecnologías es inferior al inicialmente previsto, y porque el incremento de la demanda es notablemente superior al contemplado en el Plan inicial. En él se introducen importantes modificaciones al alza de los objetivos de potencia establecidos: el objetivo de potencia fotovoltaica se amplía de $150 \mathrm{MW}$ a $400 \mathrm{MW}$ (Instituto para la Diversificación y Ahorro de la Energía, 2005).

El Real Decreto 661/20074, de 25 de mayo, da una nueva regulación a la producción de energía eléctrica en régimen especial. Con él se pretende que en el año 2010 se alcance el objetivo nacional incluido en la Directiva 2001/77/ CE $^{5}$ del Parlamento Europeo y del Consejo, de 27 de septiembre de 2001, relativa a la promoción de la electricidad generada a partir de fuentes de energía renovables en el mercado interior de la electricidad, de manera que al menos el $29,4 \%$ del consumo bruto de electricidad en 2010 provenga de fuentes de energía renovables. En su artículo 36 se recogen las tarifas y primas correspondientes a las instalaciones solares fotovoltaicas.

En septiembre de 2008, el Gobierno de España comprueba que el crecimiento de la potencia instalada experimentado por la tecnología solar fotovoltaica está siendo muy superior al esperado. Según la información publicada por la Comisión Nacional de Energía en relación al cumplimiento de los objetivos de las instalaciones del régimen especial, determinado de acuerdo con los artículos 21 y 22 del Real Decreto 661/2007, se superó el $85 \%$ del objetivo de potencia instalada fotovoltaica para 2010 y en el mes de mayo de 2008, se alcanzaron ya los $1.000 \mathrm{MW}$ de potencia instalada.

Por este motivo promulga el Real Decreto $1578 / 2008^{6}$, de 26 de septiembre, que tiene como objeto el establecimiento de un régimen económico para las instalaciones de producción de energía eléctrica de tecnología fotovoltaica a las que no les sea de aplicación los valores de la tarifa regulada previstos en el artículo 36 del Real Decreto 661/2007, por su fecha de inscripción definitiva. Las instalaciones fotovoltaicas se clasifican en dos tipos:

- Tipo I. Instalaciones que estén ubicadas en cubiertas o fachadas de construcciones dedicadas a usos residencial, de servicios, comercial o industrial, incluidas las de carácter agropecuario.

O bien, instalaciones ubicadas sobre cubiertas de aparcamiento o de sombreamiento, de áreas dedicadas a alguno de los usos anteriores, y se encuentren ubicadas en una parcela con referencia catastral urbana.

- Tipo II. Instalaciones no incluidas en el tipo I anterior.

El Real Decreto-Ley 6/20097, de 30 de abril, por el que se adoptan determinadas medidas en el sector energético y se aprueba el bono social, se justifica para el Gobierno de España por el creciente déficit tarifario, esto es, la diferencia entre la recaudación por las tarifas reguladas que fija la Administración y que pagan los consumidores por sus suministros regulados y por las tarifas de acceso que se fijan en el mercado liberalizado y los costes reales asociados a dichas tarifas.

En el Real Decreto-Ley 14/2010', de 23 de diciembre, se establecen medidas urgentes para la corrección del déficit tarifario del sector eléctrico. Para el Gobierno de España, teniendo en cuenta el ritmo de crecimiento de las instalaciones fotovoltaicas, y salvaguardando el principio de suficiencia de su retribución, por la especial incidencia que los desvíos en las previsiones de generación de esta fuente energética producen en el déficit tarifario, establece con carácter general la posibilidad de limitar las horas equivalentes de funcionamiento con derecho al régimen económico primado que tengan reconocido. En su disposición adicional primera se establece la limitación de las horas equivalentes de funcionamiento de las instalaciones fotovoltaicas en función de la zona solar climática donde se ubique la instalación de acuerdo con la clasificación

4. Boletín Oficial del Estado de 26 de mayo de 2007, $\mathrm{n}^{\circ} 126$, pp. 22.846-22.886.

5. Diario Oficial de las Comunidades Europeas de 27 de octubre de 2001, L 283-33/L 283-40.

6. Boletín Oficial del Estado de 27 de septiembre de 2008, $n^{\circ} 234$, pp. 39.117-39.125.

7. Boletín Oficial del Estado de 7 de mayo de 2009, n 111, pp. 39.404-39.419.

8. Boletín Oficial del Estado de 24 de diciembre de 2010, n 312, pp. 106.386-106.394. 
de zonas climáticas según la radiación solar media en España establecidas en el Real Decreto 314/2006 ${ }^{9}$, de 17 de marzo, por el que se aprueba el Código Técnico de la Edificación.

El 11 de noviembre de 2011, el Gobierno de España aprueba el Plan de Energías renovables 2011-2020, con el que se pretende que en 2020 España cuente con 7.250 MW de potencia solar fotovoltaica, y por tanto 3.025 MW más que en 2011 (Instituto para la Diversificación y Ahorro de la Energía, 2011)

Según Costa (2016), la rápida evolución de los costes regulados en el sistema eléctrico experimentada desde 2006 no fue compensada con un incremento a la par de las tarifas, lo que provocó la aparición de un déficit por la diferencia entre ingresos y costes del sistema que tomó la denominación de déficit de tarifa. El montante del déficit asciende en 2013 hasta los 30.000 millones de euros, representando más del 2\% del Producto Interior Bruto. Esta situación representó el inicio de una serie de modificaciones regulatorias con el fin de conseguir la sostenibilidad económica y financiera del sistema eléctrico.

Con el Real Decreto-Ley $1 / 2012^{10}$, de 27 de enero, se procede a la suspensión de los procedimientos de preasignación de retribución y a la supresión de los incentivos económicos para nuevas instalaciones de producción de energía eléctrica en régimen especial. Para el Gobierno de España, el crecimiento de las tecnologías incluidas en el régimen especial ha permitido superar con creces en 2010 los objetivos de potencia instalada previstos en el Plan de Energías Renovables 2005-2010 para la tecnología eólica y en particular para las tecnologías solar termoeléctrica y solar fotovoltaica. Además, la superación de los objetivos ha puesto de manifiesto un desequilibrio entre los costes de producción y el valor de las primas, suponiendo un incremento del sobrecoste para el sistema en concepto de primas para las tecnologías solares de más de 2.000 millones en 2010, cifra que se incrementará en 2.000 millones de euros anuales a partir de 2014.

La Ley 15/2012 ${ }^{11}$, de 27 de diciembre, de medidas fiscales para la sostenibilidad energética, tiene como objetivo armonizar el sistema fiscal español con un uso más eficiente y respetuoso con el medioambiente y la sostenibilidad, valores que inspiran esta reforma de la fiscalidad, y que están en línea con los principios básicos que rigen la política fiscal, energética, y ambiental de la Unión Europea. Además, con el fin de favorecer el equilibrio presupuestario establece un impuesto sobre el valor de la producción de la energía eléctrica, de carácter directo y naturaleza real, que grava la realización de actividades de producción e incorporación al sistema eléctrico español. El impuesto se exige al tipo del 7\%.

Ante la falta de resultados significativos para frenar el déficit de tarifa, que en el año 2013 supone 3.600 millones de euros, se promulga el Real Decreto-Ley $9 / 2013^{12}$, de 12 de julio, por el que se adoptan medidas urgentes para garantizar la estabilidad financiera del sistema eléctrico, que culmina con la Ley $24 / 2013^{13}$, de 26 de diciembre, del Sector Eléctrico y sus subsiguientes desarrollos normativos (Díaz, A.C. et al., 2015).

La Ley 24/2013 tiene como finalidad básica implantar la regulación del sector eléctrico garantizando el suministro eléctrico con los niveles necesarios de calidad y al mínimo coste posible, asegurar la sostenibilidad económica y financiera del sistema y permitir un nivel de competencia efectiva en el sector eléctrico, todo ello dentro de los principios de protección medioambiental de una sociedad moderna.

Según la Ley, el régimen retributivo de las energías renovables, cogeneración y residuos se basará en la necesaria participación en el mercado de estas instalaciones, complementado los ingresos de mercado con una retribución regulada específica que permita a estas tecnologías competir en nivel de igualdad con el resto de tecnologías en el mercado. Esta retribución específica complementaria será suficiente para alcanzar el nivel mínimo necesario para cubrir los costes que, a diferencia de las tecnologías convencionales, estas no puedan recuperar en el mercado y les permitirá obtener una rentabilidad adecuada con referencia a la instalación tipo en cada caso aplicable.

El Real Decreto 413/2014 ${ }^{14}$, de 6 de junio, regula la actividad de producción de energía eléctrica a partir de fuentes de energía renovables, cogeneración y residuos. Con este nuevo marco, las instalaciones podrán

9. Boletín Oficial del Estado de 28 de marzo de 2006, n 74, pp. 11.816-11.831.

10. Boletín Oficial del Estado de 28 de enero de 2012, n 24, pp. 8.068-8.072.

11. Boletín Oficial del Estado de 28 de diciembre, $n^{\circ} 312$, pp. 88081-88.096.

12. Boletín Oficial del Estado de 13 de julio de 2013, n 167, pp. 52.106-52147.

13. Boletín Oficial del Estado de 27 de diciembre de 2013, n 310, pp. 105.198-105.294

14. Boletín Oficial del Estado de 10 de junio de 2014, nº 140, pp. 43.876-43978. 
percibir durante su vida útil regulatoria, adicionalmente a la retribución por la venta de la energía valorada al precio del mercado, una retribución específica compuesta por un término por unidad de potencia instalada que cubra, cuando proceda, los costes de inversión para cada instalación tipo que no puedan ser recuperados por la venta de la energía en el mercado, al que se denomina retribución a la inversión, y un término a la operación que cubra, en su caso, la diferencia entre los costes de explotación y los ingresos por la participación en el mercado de producción de dicha instalación tipo, al que se denomina retribución a la operación.

En la Orden IET/1045/2014 ${ }^{15}$, de 16 de junio, se aprueban los parámetros retributivos de las instalaciones tipo aplicables a determinadas instalaciones de producción de energía eléctrica a partir de fuentes de energía renovables, cogeneración y residuos.

Los nuevos desarrollos normativos asignan un sistema automático de revisión de la tarifa en el caso de que se produzca un desajuste entre ingresos y gastos que supere el límite estipulado, para de esta forma poder controlar el volumen máximo que puede alcanzar el déficit. La nueva medida acaba con el sistema anterior, por el que la remuneración que obtenían las plantas por toda la energía que producían era a través de un sistema de primas diferentes según las tecnologías, y se sustituye por otro sistema en el que simplemente se garantiza la rentabilidad razonable que obtendría una empresa eficiente y bien gestionada a través de sus inversiones, después de haber vendido su energía en el mercado. Este cambio afecta al sistema retributivo de las nuevas instalaciones y al de las que están en funcionamiento (Costa, M.T., 2016). Por tal motivo han surgido conflictos judiciales derivados del riesgo regulatorio (Castro-Gil, J. et al., 2014) y la seguridad jurídica (García, J. et al., 2014).

El Real Decreto 900/2015 ${ }^{16}$, de 9 de octubre, regula las condiciones administrativas, técnicas y económicas de las modalidades de suministro de energía eléctrica con autoconsumo y de producción con autoconsumo. Esta norma queda derogada con efectos de 7 de abril de 2019.

Con el Real Decreto-Ley 15/2018 17 , de 5 de octubre, se adoptan medidas urgentes para la transición energética y la protección de los consumidores, y tiene por objeto el establecimiento de las condiciones administrativas, técnicas y económicas para las modalidades de autoconsumo de energía eléctrica definidas en el artículo 9 de la Ley 24/2013 del Sector Eléctrico.

El Real Decreto 244/201918, de 5 de abril, regula las condiciones administrativas, técnicas y económicas del autoconsumo de energía eléctrica. Da continuidad a lo establecido en el Real Decreto-Ley 15/2018, y reglamenta todos los aspectos no definidos en dicho texto. Clasifica y define las distintas clases de autoconsumo, consagrando la figura del autoconsumo colectivo, para que varios consumidores puedan asociarse a una misma planta de generación.

Según Fernández-Cuesta (2016), los casi veinte años transcurridos desde la promulgación de la Ley del Sector Eléctrico de 1997 se han caracterizado por la tensión permanente entre el impulso liberalizador y la pretensión de los agentes del sector de garantizar sus márgenes, en ocasiones con el amparo de los poderes públicos, ávidos de recaudación fiscal en el caso autonómico o simplemente temerosos de la pérdida de control o poder que una auténtica liberalización supondría".

\subsection{Evolución de la potencia instalada y de la producción de electricidad con energía solar fotovoltaica}

La energía solar fotovoltaica es la segunda tecnología renovable con mayor desarrollo en España. Al finalizar el año 2018 aporta el 4,5\% de la potencia eléctrica instalada en el conjunto del país, y el 10,2\%de la electricidad generada con renovables. Detrás de estos datos se encuentran dos hechos a tener en cuenta. El primero es que España ha sido pionera en el Planeta en la implantación de instalaciones fotovoltaicas. El segundo, y no menos importante, es que ésta ha sido posible por la implicación de decenas de miles de inversores ante

15. Boletín Oficial del Estado de 20 de junio de 2014, n 150, pp. 46.430-48.190.

16. Boletín Oficial del Estado de 10 de octubre de 2015, n² 243, pp. 94.874-94.917.

17. Boletín Oficial del Estado de 6 de octubre de 2018, n² 242, pp. 97.430-97.467.

18. Boletín Oficial del Estado de 6 de abril de 2019, n 83 , pp. 35.674-35.719. 
el decidido impulso de este sector energético por parte del Gobierno de España, a través de unos incentivos económicos muy atractivos, como se demuestra en el apartado anterior.

Los promotores impulsaron plantas fotovoltaicas de hasta $5 \mathrm{kWp}$ que se reunían en parques de mucha mayor potencia lo cual, por una parte, garantizaba obtener la máxima retribución a sus titulares y, por la otra, se aprovechaban de economías de escala dado que los sistemas de conexión, los equipos de control del perímetro del recinto, los accesos a la instalación, etc. eran comunes. El sector fotovoltaico, de este modo, ganó experiencia en el montaje de plantas en el suelo que resultaban de la yuxtaposición de los denominados huertos solares (Mir, P., 2012).

La Comisión Nacional de Energía define un huerto fotovoltaico como "el conjunto de instalaciones individuales que utilizan esta misma tecnología, con potencias individuales no superior a $100 \mathrm{~kW}$ y compartiendo todas ellas, directa o indirectamente, un mismo punto frontera con la red de transporte o de distribución. En los huertos fotovoltaicos se puede liquidar globalmente la energía generada cuando exista un mismo representante para todos los titulares de las instalaciones que forman parte del huerto, considerándose la energía medida en el punto frontera con la red. Los huertos fotovoltaicos que totalizan una potencia global superior a $10 \mathrm{MW}$ deben estar adscritos a un centro de control de generación, que actúa como interlocutor con el operador del sistema. Cuando estos huertos se encuentran situados en los sistemas aislados y Extrapeninsulares, dicha obligación de adscripción lo es para los huertos de potencia global superior a 1 MW "(Comisión Nacional de Energía, 2008).

La evolución del número de instalaciones solares fotovoltaicas en España, de la potencia instalada y de la energía vendida durante los últimos veinte años queda recopilada en la tabla 9.

El mayor impulso para la implantación de las instalaciones fotovoltaicas viene dado por el incremento de los incentivos del Real Decreto 661/2007, sobre todo en lo referente a las atractivas tarifas para los promotores de instalaciones fotovoltaicas $(0,440381 € / \mathrm{kWh}$, es decir, el $575 \%$ de la tarifa media de referencia ese año). Sus consecuencias se reflejan en estos datos: en abril de 2008 se alcanzan los 1.000 MW y en septiembre de ese año se superan los 3.000 MW. Este "boom" de instalaciones y potencia se debe a su consideración como producto de inversión financiera por pequeños inversores nacionales y grandes inversores nacionales e internacionales (Sevilla, M. et al., 2013).

Este espectacular desarrollo lleva a algunos autores a denominar el fenómeno como una "burbuja". Para la Fundación de Estudios sobre la Energía, el efecto llamada del Real Decreto 661/2007, que en el caso de la fotovoltaica llegaba a cifras verdaderamente asombrosas ( 12 veces superiores a las primas de la eólica, por poner una referencia relativa), provocó un efecto llamada que produjo las burbujas solares, fotovoltaica y termosolar (Fundación de Estudios sobre la Energía, 2010).

Mir (2012) describe este proceso en detalle "Durante el otoño de 2007 y el posterior invierno, la capacidad instalada empezó a crecer, aunque de forma un tanto irregular. Sin embargo, habida cuenta del lapsus necesario para su tramitación y ejecución, es de imaginar que ya entonces un número cada vez mayor de proyectos fotovoltaicos estaban entrando en las oficinas de las diversas administraciones regionales para su aprobación. Por consiguiente, había indicios de que la actividad fotovoltaica empezaba a expandirse y lo hacía de forma cada vez más rápida. Tales proyectos en curso fueron los que se materializaron en la primavera y verano de 2008. Entre los meses de julio y septiembre se llegaron a instalar más de $500 \mathrm{MW}$ mensuales. Los más de $1.000 \mathrm{MW}$ existentes en abril de 2008 , se habían más que duplicado tan solo cuatro meses después. En agosto, los analistas pronosticaban que, para fin de año se alcanzarían los $3.000 \mathrm{MW}$. En realidad, a finales de septiembre había ya inscritos 3.105 MW. En los nueve primeros meses de 2008, se instalaron más de las cuatro quintas partes de toda la capacidad de generación fotovoltaica hasta entonces acumulada. Si se consideran los 16 meses de vigencia del Real Decreto 661/2007 para la técnica fotovoltaica, la tasa de crecimiento mensual de la capacidad instalada fue un increíble 15,17\%. El boom que estaba viviendo el sector fotovoltaico español carecía de precedentes y dejaba boquiabierto al mundo entero".

Para Gómez et al. (2016), las causas de la burbuja fotovoltaica son responsabilidad incuestionable del Gobierno de España. Los costes de la electricidad fotovoltaica se evaluaron erróneamente para dimensionar las primas, los costes futuros de las tecnologías se proyectaron incorrectamente y el impacto en el sistema eléctrico se infravaloró. Se ignoró negligentemente el objetivo de $400 \mathrm{MW}$ fotovoltaicos fijado en el Plan de 
Tabla 9. Instalaciones fotovoltaicas, potencia instalada(MW) y producción de electricidad (GWh). $1998-2018$.

\begin{tabular}{|c|c|c|c|c|}
\hline Año & Número de instalaciones & Potencia instalada MW & $\begin{array}{l}\text { Energía vendida } \\
\text { GWh }\end{array}$ & $\begin{array}{c}\text { \% sobre generación } \\
\text { renovable }\end{array}$ \\
\hline 1998 & 12 & 1 & 1 & - \\
\hline 1999 & 16 & 2 & 1 & - \\
\hline 2000 & 45 & 2 & 1 & - \\
\hline 2001 & 196 & 4 & 2 & - \\
\hline 2002 & 795 & 7 & 4 & - \\
\hline 2003 & 1.581 & 11 & 9 & - \\
\hline 2004 & 3.266 & 23 & 17 & - \\
\hline 2005 & 5.391 & 47 & 38 & - \\
\hline 2006 & 9.874 & 146 & 99 & - \\
\hline 2007 & 20.284 & 690 & 473 & 0,8 \\
\hline 2008 & 51.308 & 3.398 & 2.503 & 4,1 \\
\hline 2009 & 52.102 & 3.391 & 6.073 & 8,2 \\
\hline 2010 & 54.943 & 3.829 & 6.401 & 6,7 \\
\hline 2011 & 57.780 & 4.232 & 7.256 & 8,6 \\
\hline 2012 & 59.935 & 4.522 & 8.016 & 9,6 \\
\hline 2013 & 60.920 & 4.645 & 8.279 & 7,6 \\
\hline 2014 & 61.040 & 4.654 & 8.185 & 7,6 \\
\hline 2015 & 61.225 & 4.666 & 8.224 & 8,8 \\
\hline 2016 & 61.228 & 4.673 & 7.971 & 7,9 \\
\hline 2017 & 61.350 & 4.678 & 8.394 & 9,9 \\
\hline 2018 & 63.364 & 4.698 & 7.752 & 10,2 \\
\hline
\end{tabular}

Fuente: Comisión Nacional de los Mercados y la Competencia, y Red Eléctrica de España.

Energías Renovables 2005-2010. La delirante meta, frecuentemente declarada, de posicionar a España como líder en el despliegue de las energías renovables probablemente contribuyó a obnubilar a los políticos, eclipsar los riesgos y animar la inversión especulativa.

Se había producido un crecimiento del 500\% (el mayor en todo el mundo) en tan sólo un año. Según Mir (2012), varios factores habían favorecido esta evolución explosiva: (a) ya habían aparecido los primeros síntomas de la crisis financiera, así que muchos inversores estaban buscando productos financieros alternativos; (b) el crédito era barato y fácil de conseguir; (c) el marco legal permitía construir grandes instalaciones compartidas por muchos pequeños propietarios que compartían servicios (granjas solares); (d) la evidencia de que las condiciones empeorarían después de la fecha límite; (e) la disponibilidad de mucho sol y tierras; y (f) el cambio del euro con respecto al dólar, que favorecía la importación de módulos fotovoltaicos. El asombroso crecimiento del sector fotovoltaico español, especialmente en 2008, convirtió a España en el segundo país con más potencia instalada en el Planeta, después de Alemania (De la Hoz, J. et al., 2010; Girard, E.J. et al., 2016). 
Con el objetivo de llevar un mayor control de las nuevas instalaciones y cerrar el cupo del Real Decreto 661/2007, el Gobierno de España publica el Real Decreto 1578/2008 tan solo un año después. Su puesta en vigor produce un efecto notable en el 2009, en el que sólo se instalan 17 MW. Este Real Decreto cambió además la clasificación de las instalaciones. Si hasta entonces se clasificaban por potencia, a partir de esta normativa se realiza mediante el lugar de instalación (tejado pequeño, tejado grande o suelo). El nuevo sistema impone un registro de pre-asignación que abre cuatro convocatorias al año y un ritmo de reducción del 10 \% por año. Este nuevo Real Decreto paralizó el mercado interior, que desde entonces ha crecido sólo ligeramente hasta alcanzar la capacidad instalada al finalizar 2018de 4.698 MW, según la Comisión Nacional de los Mercados y la Competencia.

Según Unión Española Fotovoltaica (UNEF) en esa cifra no está recogida la potencia aislada y parte de la potencia de autoconsumo. Según los datos registrados por UNEF, en 2018 se instalaron en España 261,7 MW de nueva potencia fotovoltaica, lo que supone un incremento del $94 \%$ respecto a los $55 \mathrm{MW}$ registrados en 2016 y los 49 MW de 2015. De esos 261,7 MW, un 90\% (235,7 MW) corresponden al autoconsumo energético, siendo un $25 \%$ de estos relativos a autoconsumo conectado a la red para uso agrícola y $26 \mathrm{MW}$ a plantas solares sobre suelo ${ }^{19}$.

A 31 de julio de 2019, Red Eléctrica de España, a través de su Serie Estadística del Sistema Eléctrico Español, contabiliza 5.570 MW, 1.104 MW más de los 4.466 MW que tiene registrados al finalizar el año 2018. En tan solo los siete meses transcurridos de 2019 se ha incrementado en una cuarta parte la capacidad de generación eléctrica con esta tecnología. Son las consecuencias del desarrollo de las plantas de autoconsumo y de las dedicadas a la producción de electricidad para su comercialización.

Según EurObserv'ER, España cuenta con una potencia en energía solar fotovoltaica al finalizar el año 2018 de 4.751 MW, que lo sitúa en quinto lugar de los países de la Unión Europea (Tabla 10) y en el duodécimo a nivel mundial (Tabla 11).

En el caso de China, el 80\% de la fotovoltaica instalada en 2018 fue distribuida. En 2017, de un total de 130.000 MW acumulados, 26.000 eran en potencia distribuida. Los proyectos de generación distribuida son gestionados directamente por los gobiernos locales (García, 2019).

\subsection{Localización de la potencia instalada y de la producción de electricidad con energía solar fotovoltaica}

Al finalizar el año 2018 cinco Comunidades Autónomas concentran la mayor parte de la potencia y dela producción de electricidad solar fotovoltaica en España (Tablas 12 y 13).En Castilla-La Mancha se localiza una quinta parte de la potencia y de la producción. Le sigue en capacidad y producción Andalucía, que aporta al total nacional el $19 \%$ y $18,7 \%$ respectivamente. Extremadura es la tercera región con una contribución del 13,1\% y 12,1\%; cifras próximas a las de Castilla y León con el 10,5 y 10,4\% respectivamente. Por último, destaca la Región de Murcia que, a pesar de estar constituida por una sola provincia, cuenta con el 9,5\% de la potencia y contribuye con el 9,4\% de la producción eléctrica. Estas cinco regiones acumulan el 70,2\% de la capacidad instalada y el $72,5 \%$ de la producción.

También se ha reflejado en las tablas 12 y 13 la aportación de la energía solar fotovoltaica al conjunto de las energías renovables y al total de tecnologías, tanto en lo referente a potencia como a producción de electricidad.

En Andalucía, su clima y el entorno orográfico del sur de España le otorgan un enorme potencial para la instalación de centrales fotovoltaicas conectadas a redes eléctricas y también para el uso individual (Arán et al., 2008). Además, Andalucía se ha caracterizado por contar con un marco regulatorio y de planificación muy estable, con objetivos claramente orientados hacia un crecimiento de las energías renovables y un desarrollo importante de las redes eléctricas y de gas, en línea con los objetivos europeos en materia energética (González, N., 2015). La región dispone de abundante espacio en las zonas rurales del interior.

19. https://unef.es/2019/02/la-energia-fotovoltaica-en-espan\%CC\%83a-avanza-de-forma-decidida-en-2018-con-un-crecimientodel-94-de-la-potencia-instalada/. 
Tabla 10. Potencia de energía solar fotovoltaica instalada (MW), producción de electricidad de origen fotovoltaico (GWh) y potencia por habitante (W). Países de la Unión Europea. 31-12-2018.

\begin{tabular}{|c|c|c|c|c|c|}
\hline & \multicolumn{2}{|c|}{ Potencia } & \multicolumn{2}{|c|}{ Producción } & \multirow{2}{*}{$\begin{array}{c}\text { Potencia/habi- } \\
\text { tante } \\
\text { W }\end{array}$} \\
\hline & MW & \% U.E & GWh & \% U.E. & \\
\hline Alemania & 45.277 & 39,53 & 46.164 & 37,74 & 546,9 \\
\hline Italia & 20.107 & 17,55 & 22.654 & 18,52 & 332,4 \\
\hline Reino Unido & 13.054 & 11,40 & 12.922 & 10,56 & 197,8 \\
\hline Francia & 9.466 & 8,26 & 10.196 & 8,34 & 141,4 \\
\hline España & 4.751 & 4,15 & 7.785 & 6,36 & 101,8 \\
\hline Países Bajos & 4.300 & 3,75 & 3.152 & 2,58 & 250,3 \\
\hline Bélgica & 4.255 & 3,71 & 3.563 & 2,91 & 373,2 \\
\hline Grecia & 2.652 & 2,31 & 3.791 & 3,10 & 246,9 \\
\hline Rep. Checa & 2.049 & 1,79 & 2.340 & 1,91 & 193,0 \\
\hline Austria & 1.433 & 1,25 & 1.433 & 1,17 & 162,4 \\
\hline Rumanía & 1.377 & 1,20 & 1.860 & 1,52 & 70,5 \\
\hline Bulgaria & 1.036 & 0,90 & 1.404 & 1,15 & 146,9 \\
\hline Dinamarca & 1.002 & 0,87 & 953 & 0,78 & 173,3 \\
\hline Hungría & 754 & 0,66 & 765 & 0,63 & 77,1 \\
\hline Portugal & 671 & 0,59 & 1.020 & 0,83 & 65,2 \\
\hline Eslovaquia & 531 & 0,46 & 509 & 0,42 & 97,6 \\
\hline Polonia & 487 & 0,42 & 300 & 0,25 & 12,8 \\
\hline Suecia & 424 & 0,37 & 400 & 0,33 & 41,9 \\
\hline Eslovenia & 256 & 0,22 & 294 & 0,24 & 123,9 \\
\hline Luxemburgo & 134 & 0,12 & 110 & 0,09 & 222,6 \\
\hline Malta & 131 & 0,11 & 183 & 0,15 & 276,0 \\
\hline Finlandia & 125 & 0,11 & 162 & 0,13 & 22,7 \\
\hline Chipre & 113 & 0,10 & 177 & 0,14 & 130,9 \\
\hline Lituania & 74 & 0,06 & 80 & 0,07 & 26,3 \\
\hline Croacia & 61 & 0,05 & 80 & 0,07 & 14,9 \\
\hline Irlanda & 29 & 0,03 & 20 & 0,02 & 6,8 \\
\hline Letonia & 1 & 0,00 & 1 & 0,00 & 0,5 \\
\hline Estonia & 0 & 0,00 & 0 & 0,00 & 0 \\
\hline Total & 114.549 & 100,00 & 122.318 & 100,00 & 223,6 \\
\hline
\end{tabular}

Fuente: EurObserv’ER.BaromètrePhotovoltaïque 2019. 
Tabla 11. Países del Planeta con más potencia fotovoltaica instalada. 31-12-2018. MW.

\begin{tabular}{|c|c|c|}
\hline & MW & $\%$ \\
\hline China & 175.131 & 34,39 \\
\hline Estados Unidos & 62.127 & 12,20 \\
\hline Japón & 55.851 & 10,97 \\
\hline Alemania & 45.277 & 8,89 \\
\hline India & 27.347 & 5,37 \\
\hline Italia & 20.107 & 3,95 \\
\hline Reino Unido & 13.054 & 2,56 \\
\hline Australia & 12.560 & 2,47 \\
\hline Francia & 9.466 & 1,86 \\
\hline Corea del Sur & 7.742 & 1,52 \\
\hline Turquía & 5.062 & 0,99 \\
\hline España & 4.751 & 0,93 \\
\hline Países Bajos & 4.300 & 0,84 \\
\hline México & 3.580 & 0,70 \\
\hline Taiwan & 2.739 & 0,54 \\
\hline Grecia & 2.652 & 0,52 \\
\hline Brasil & 2.346 & 0,46 \\
\hline República Checa & $2 . .049$ & 0,40 \\
\hline Otros países & 53.159 & 10,44 \\
\hline Total mundial & 509.300 & 100,00 \\
\hline
\end{tabular}

Fuente: SolarPower Europe. Global Market Outlook.For Solar Power / 2019-2023.

También ha facilitado la implantación de instalaciones fotovoltaicas extensivas el bajo precio de la tierra y el mayor rendimiento ofrecido por la energía fotovoltaica en comparación con los usos agrícolas tradicionales (Mérida, M. et al., 2015). La componente territorial del modelo energético renovable en esta Región está marcada por la dispersión de instalaciones (Prados, M.J., 2010). Las centrales se distribuyen espacialmente en el Valle del Guadalquivir y en las Ilanuras fértiles y las mesetas entre las cordilleras béticas (Mérida, M. et al., 2015). No obstante, hay que tener en cuenta que un criterio esencial para su ubicación es la proximidad a las principales redes de distribución de electricidad, ya que son imprescindibles para verter y distribuir la electricidad generada por las instalaciones (Díaz, M.P. et al., 2011; Díaz, M.P. et al., 2017).

Según Baraja y Herrero (2010), Castilla y León ha tenido un protagonismo indiscutible en el proceso "explosivo" de implantación de la energía eléctrica de origen fotovoltaico. A ello ha contribuido la apuesta de la Administración Autonómica por este tipo de energía. Este proceso de implantación presenta rasgos de una notable singularidad geográfica. La energía fotovoltaica está claramente asociada a las llanuras del interior de la región. Estas preferencias responden a una lógica territorial asociada a un cambio de modelo en la forma de inserción de los espacios económicos y a unos intereses concretos (Baraja, E. y Luque, D., 2010). 
Tabla 12. Potencia eléctrica instalada en energía solar fotovoltaica, en energías renovables y en total (MW). Aportación de la potencia fotovoltaica a la potencia de energías renovables y a la potencia total. Comunidades Autónomas. 2018

\begin{tabular}{|c|c|c|c|c|c|}
\hline $\begin{array}{l}\text { Comunidad } \\
\text { Autónoma }\end{array}$ & $\begin{array}{c}\text { Potencia } \\
\text { Fotovoltaica MW }\end{array}$ & $\begin{array}{c}\text { Potencia Renovables } \\
\text { MW }\end{array}$ & $\begin{array}{c}\text { Potencia Total } \\
\text { MW }\end{array}$ & $\begin{array}{l}\text { \% Fotovoltaica/ } \\
\text { Renovables }\end{array}$ & $\begin{array}{c}\text { \% Fotovoltaica/ } \\
\text { Total }\end{array}$ \\
\hline Castilla-La Mancha & 925 & 5.521 & 8.204 & 16,75 & 11,27 \\
\hline Andalucía & 882 & 5.030 & 15.486 & 17,53 & 5,70 \\
\hline Extremadura & 564 & 2.878 & 5.762 & 19,60 & 9,79 \\
\hline Castilla y León & 496 & 10.533 & 13.584 & 4,71 & 3,65 \\
\hline Región de Murcia & 442 & 754 & 4.373 & 58,62 & 10,11 \\
\hline $\begin{array}{l}\text { Comunidad Valen- } \\
\text { ciana }\end{array}$ & 361 & 2.219 & 8.224 & 16,27 & 4,39 \\
\hline Cataluña & 269 & 3.545 & 11.874 & 7,59 & 2,27 \\
\hline Aragón & 169 & 3.518 & 7.233 & 4,80 & 2,34 \\
\hline Canarias & 167 & 597 & 2.997 & 27,97 & 5,57 \\
\hline Navarra & 162 & 1.462 & 2.833 & 11,08 & 5,72 \\
\hline La Rioja & 86 & 590 & 1.398 & 14,58 & 6,15 \\
\hline Baleares & 81 & 124 & 2.285 & 65,32 & 3,54 \\
\hline $\begin{array}{l}\text { Comunidad de } \\
\text { Madrid }\end{array}$ & 64 & 232 & 458 & 27,59 & 13,97 \\
\hline País Vasco & 27 & 455 & 2.906 & 5,93 & 0,93 \\
\hline Galicia & 17 & 7.239 & 10.981 & 0,23 & 0,15 \\
\hline Cantabria & 2 & 154 & .815 & 1,30 & 0,25 \\
\hline Asturias & 1 & 1.415 & 4.512 & 0,07 & 0,02 \\
\hline Total España & 4.715 & 46.266 & 103.925 & 10,19 & 4,54 \\
\hline
\end{tabular}

Fuente: Red Eléctrica de España.

\section{MEDIDAS PARA EL IMPULSO DE LA ENERGÍA SOLAR FOTOVOLTAICA EN ESPAÑA}

\subsection{Marco Legal para el autoconsumo eléctrico}

El Real Decreto 900/2015 informa que los consumidores que realizan autoconsumo abonarán los peajes de acceso a las redes de transporte y distribución como contribución a la cobertura de los costes de dichas redes y serán abonados por el uso real que se realiza de ellas, es decir por la potencia contratada y la energía medida en el punto frontera asociado a ella.

Clasifica y define las distintas clases de autoconsumo y de producción con autoconsumo. Quedan exentas del pago del peaje las instalaciones aisladas, las de menos de 10 kW, y las situadas en Canarias, Ceuta y Melilla.

El Real Decreto-Ley 15/2018 deroga el anterior. Presenta dos novedades: a) la energía autoconsumida de origen renovable, cogeneración o residuos queda exenta de todo tipo de cargas y peajes; b) las instalaciones de producción no superiores a $100 \mathrm{~kW}$ de potencia asociadas a modalidades de suministro de autoconsumo 
con excedentes estarán exentas de la obligación de inscripción en el registro administrativo de instalaciones de producción eléctrica.

Tabla 13. Producción de electricidad con energía solar fotovoltaica, con energías renovables y en total (GWh). Aportación de la solar fotovoltaica a la producción con energías renovables y con la potencia total. Comunidades Autónomas. 2018

\begin{tabular}{|c|c|c|c|c|c|}
\hline $\begin{array}{l}\text { Comunidad } \\
\text { Autónoma }\end{array}$ & $\begin{array}{l}\text { Producción con } \\
\text { Energía } \\
\text { Fotovoltaica } \\
\text { GWh }\end{array}$ & $\begin{array}{c}\text { Producción con } \\
\text { Energías Reno- } \\
\text { vables } \\
\text { GWh }\end{array}$ & $\begin{array}{c}\text { Producción } \\
\text { Energética } \\
\text { Total } \\
\text { GWh }\end{array}$ & $\begin{array}{c}\text { \% } \\
\text { Producción } \\
\text { Fotovoltaica/ } \\
\text { Producción } \\
\text { Renovables }\end{array}$ & $\begin{array}{c}\text { \% Producción } \\
\text { Fotovoltaica/ } \\
\text { Producción } \\
\text { Total }\end{array}$ \\
\hline Castilla-La Mancha & 1.579 & 10.690 & 21.670 & 14,77 & 7,29 \\
\hline Andalucía & 1.470 & 9.814 & 34.265 & 14,98 & 4,29 \\
\hline Extremadura & 1.019 & 3.568 & 20.981 & 28,56 & 4,86 \\
\hline Castilla y León & 803 & 20.593 & 26.796 & 3,90 & 3,00 \\
\hline Región de Murcia & 743 & 1.366 & 6.089 & 54,39 & 12,20 \\
\hline Comunidad Valenciana & 527 & 4.410 & 19.122 & 11,95 & 2,76 \\
\hline Cataluña & 384 & 8.812 & 42.468 & 4,36 & 0,90 \\
\hline Navarra & 296 & 3.611 & 5.181 & 8,20 & 5,71 \\
\hline Aragón & 288 & 8.699 & 15.207 & 3,31 & 1,89 \\
\hline Canarias & 272 & 929 & 8.841 & 29,28 & 3,08 \\
\hline La Rioja & 130 & 1.213 & 2.393 & 10,72 & 5,43 \\
\hline Baleares & 113 & 254 & 4.820 & 44,49 & 2,34 \\
\hline Comunidad de Madrid & 85 & 441 & 1.251 & 19,27 & 6,79 \\
\hline País Vasco & 28 & 1.176 & 5.142 & 2,38 & 0,54 \\
\hline Galicia & 18 & 17.396 & 31.312 & 0,10 & 0,06 \\
\hline Cantabria & 2 & 798 & 2.213 & 0,25 & 0,09 \\
\hline Asturias & 0 & 3.607 & 12.804 & 0,00 & 0,00 \\
\hline Total España & 7.757 & 97.377 & 260.555 & 7,97 & 2,98 \\
\hline
\end{tabular}

Fuente: Red Eléctrica de España.

El Real Decreto 244/2019 clasifica y define las distintas clases de autoconsumo, consagrando la figura del autoconsumo colectivo, para que varios consumidores puedan asociarse a una misma planta de generación. Define el concepto de "instalación de producción próxima a las de consumo y asociadas a las mismas". Esto permite realizar autoconsumo tanto en instalaciones de generación situadas en la misma vivienda como en otras que estén ubicadas en las proximidades. Otra de sus novedades consiste en la implantación de un mecanismo simplificado de compensación de excedentes, es decir, de aquella energía generada por instalaciones de autoconsumo y que el usuario no consume instantáneamente. Con este Real Decreto, la comercializadora de energía compensará al usuario por la energía excedentaria en cada factura mensual. La norma abre también la posibilidad de que todas las comercializadoras puedan ofrecer servicios de autoconsumo renovable; y reduce también los trámites administrativos para los usuarios. 
En cuanto al régimen económico se establecen varias posibilidades en función del tipo de autoconsumo:

- Autoconsumo con excedentes acogidos a compensación. Pueden vender la energía en el pool, o compensar mensualmente excedentes, mediante la valoración de la energía horaria excedentaria (compensación simplificada). En importe a compensar nunca podrá exceder de la valoración mensual de la energía horaria consumida.

- Autoconsumo con excedentes no acogidos a compensación. Deben vender los excedentes en el mercado.

Para la Unión Española Fotovoltaica, este Real Decreto, "sitúa al ciudadano en el centro del modelo energético permitiéndole tener acceso libre la producción y venta de la energía y lo hace más responsable de su consumo, en línea con los principios establecidos por el paquete de medidas "Energía Limpia para todos los Europeos", y con la consecución del Acuerdo de París"20. Desde la Alianza por el Autoconsumo, se considera que la producción de energía eléctrica renovable debería tener un importante desarrollo en nuestro país, debido a su alto potencial de recurso renovable, gracias a su envidiable ubicación geográfica y su liderazgo empresarial en materia renovable ${ }^{21}$.

La tabla 14 recopila el número de instalaciones de autoconsumo con energía fotovoltaica censadas a finales de 2018, así como la potencia instalada y por Comunidades Autónomas. Según la Asociación Nacional de Productores de Energía Fotovoltaica (2019) durante el año 2018 se ha producido un incremento en el número de instalaciones del $80 \%$ respecto a las existentes en 2017, y el aumento de la potencia instalada ha sido del 40\%, lo que indica que la mayor parte de las puestas en marcha en 2018 eran de pequeño tamaño.

El 8 de agosto de 2019 el Instituto para la Diversificación y Ahorro de la Energía publica dos guías completamente actualizadas para facilitar a la ciudadanía la tramitación y puesta en marcha de instalaciones de autoconsumo: Guía para convertirse en autoconsumidos en 5 pasos y Guía profesional de tramitación del autoconsumo ${ }^{22}$.

\subsection{Las subastas de energías renovables}

Para cumplir con el objetivo europeo de que en 2020 la energía renovable aporte el 20\% al consumo de energía final, el Gobierno de España decide en 2017 potenciar el incremento de la capacidad renovable en el sistema eléctrico. Para ello establece subastas de potencia renovable en las que participan las distintas tecnologías en concurrencia competitiva con el fin de introducir los proyectos más eficientes en costes.

El sistema de subastas establecido en España se basa en procedimiento de concurrencia competiviva por el cual, las instalaciones adjudicatarias tendrán derecho a la percepción del regimen retributivo específico, complementario a los ingresos por la venta de energía en el mercado mayorista de electricidad. Este régimen retributivo específico debe permitir a los beneficiarios obtener una renta razonable que se calcula a partir del rendimiento de las Obligaciones del Estado a 10 años en el mercado secundario, incrementada en 300 puntos básicos.

El objeto de la subasta se basa en la potencia a instalar y no en el volumen de energía que las plantas adjudicatias puedan verter a la red. Hasta la fecha se hna celebrado tres subastas. La primera, celebrada en 2016, se limitó únicamente a tecnologías eólica y biomasa, mientras que en las dos siguientes se permitió el acceso a todas las tecnologías de generación renovable.

En la segunda subasta, celebrada el 17 mayo de 2017, se adjudican $3.000 \mathrm{MW}$, y de ellos solo uno a instalaciones fotovoltaicas ${ }^{23}$. En cambio, en la tercera subasta, de 27 de julio de 2017, de los $5.037 \mathrm{MW}$ adjudicados, $3.909 \mathrm{MW}$ fueron para instalaciones fotovoltaicas repartidas entre 30 empresas $^{24}$, y de ellas destaca el Grupo Cobra ${ }^{25}$, que cuenta con el 39,6\% del total (Tabla 15).

20. https://unef.es/2019/04/el-rd-situa-al-ciudadano-en-el-centro-del-modelo-energetico-al-garantizar-el-libre-acceso-a-la-energia/.

21. http://unef.es/wp-content/uploads/dlm_uploads/2017/05/alianza-por-el-autoconsumo--manifiesto-final-11-05-2017.pdf.

22. Están disponibles en: https://www.idae.es/publicaciones.

23. Boletín Oficial del Estado de 26 de mayo de 2017, nº 125, pp. 42.785-42.788.

24. Boletín Oficial del Estado de 28 de julio de 2017, n 179, pp. 70.285-70.289.

25. http://www.grupocobra.com. 
Tabla 14. Número de instalaciones de autoconsumo fotovoltaico y potencia instalada. Comunidades Autónomas. 3112-2018.

\begin{tabular}{|c|c|c|c|c|c|}
\hline \multirow{2}{*}{ Comunidad Autónoma } & \multicolumn{2}{|c|}{ Instalaciones } & \multicolumn{2}{|c|}{ Potencia instalada } & \multirow{2}{*}{$\begin{array}{l}\text { Tamaño medio } \\
\text { explotaciones kW }\end{array}$} \\
\hline & Número & \% total de España & $\mathrm{kW}$ & \% total de España & \\
\hline Cataluña & 299 & 25,93 & 6.330 & 23,40 & 21,17 \\
\hline Andalucía & 269 & 23,33 & 4.761 & 17,60 & 17,70 \\
\hline Galicia & 87 & 7,55 & 5.888 & 21,76 & 67,68 \\
\hline Comunidad de Madrid & 84 & 7,29 & 1.236 & 4,57 & 14,71 \\
\hline Región de Murcia & 72 & 6,24 & 1.180 & 4,36 & 16,39 \\
\hline Castilla-La Mancha & 57 & 4,94 & 732 & 2,71 & 12,84 \\
\hline Baleares & 53 & 4,60 & 1.364 & 5,04 & 25,74 \\
\hline Comunidad Valenciana & 49 & 4,25 & 823 & 3,04 & 16,80 \\
\hline Canarias & 40 & 3,47 & 1.190 & 4,40 & 29,75 \\
\hline Navarra & 37 & 3,21 & 728 & 2,69 & 19,68 \\
\hline Castilla y León & 34 & 2,95 & 1145 & 4,23 & 33,68 \\
\hline Aragón & 19 & 1,65 & 921 & 3,40 & 48,47 \\
\hline País Vasco & 14 & 1,21 & 424 & 1,57 & 30,29 \\
\hline Asturias & 13 & 1,13 & 142 & 0,52 & 10,92 \\
\hline Extremadura & 13 & 1,13 & 82 & 0,30 & 6,31 \\
\hline La Rioja & 9 & 0,78 & 71 & 0,26 & 7,89 \\
\hline Cantabria & 3 & 0,26 & 15 & 0,06 & 5,00 \\
\hline Ceuta & 1 & 0,09 & 24 & 0,09 & 24,00 \\
\hline Melilla & 0 & 0,00 & 0 & 0,00 & 0,00 \\
\hline Total & 1.153 & 100,00 & 27.058 & 100,00 & 23,47 \\
\hline
\end{tabular}

Fuente: Asociación Nacional de Productores de Energía Fotovoltaica (ANPIER).Anuario Fotovoltaico 2019.

Frente a los macroproyectos de las grandes empresas, es reseñable del caso de la Sociedad Sinlimitsol, S.L., adjudicataria de una potencia de $2 \mathrm{MW}$, e integrada por 75 familias que suman un total de 100 participaciones de 20.000 euros, y que ha inaugurado su instalación en el municipio murciano de Yecla a comienzos de $2019^{26}$.

Según el "Informe de seguimiento de grandes plantas fotovoltaicas en España. $2^{\text {a }}$ Versión. Diciembre de 2018", las empresas que resultaron adjudicatarias del concurso han realizado un enorme esfuerzo para cumplir con los compromisos de dicha subasta, y están cumpliendo con sus obligaciones, para así lograr el objetivo de que los 3.909 MW estén funcionando antes del año 2020 (Hernández, C.et al., 2019).

26. https://www.eldiario.es/ballenablanca/transicion_energetica/Terminada-Murcia-adjudicada-subasta-particulares_0_859864660.html. 
Tabla 15.Potencia fotovoltaica adjudicada en la Subasta de Energías Renovables del 27 de julio de 2017.

\begin{tabular}{|c|c|}
\hline Nombre del adjudicatario & Potencia adjudicada. kW \\
\hline Cobra Concesiones, S.L. & 1.550 .000 \\
\hline X-Elio Energy, S.L. & 455.000 \\
\hline Enel Green Power España, S.L. & 338.670 \\
\hline Desarrollos Fotovoltaicos Meridionales & 316.000 \\
\hline Gas Natural Fenosa Renovables & 250.000 \\
\hline Planta FV3, S.L. & 250.000 \\
\hline Otras Producciones de Energía Fotovoltaica, S.L. & 200.000 \\
\hline Lotapera, S.L. & 182.500 \\
\hline Grupo Tec Servicios Avanzados, S.A. & 91.666 \\
\hline Alter Enersun, S.A. & 50.000 \\
\hline Engie España, S.L.U. & 50.000 \\
\hline Monegros Solar, S.A. & 50.000 \\
\hline GestampEolica, S.L. & 24.000 \\
\hline Fres Wind Moon Systems, S.L. & 23.750 \\
\hline Rios Renovables, S.L.U. & 21.000 \\
\hline Operating Business 2, S.L. & 20.000 \\
\hline Alten El Casar, S.L. & 13.020 \\
\hline Jorge Energy, S.L. & 9.400 \\
\hline Climaster Solar Fv, S.L. & 3.911 \\
\hline Sinlimitsol, S.L. & 2.000 \\
\hline Dalar Solar, S.L.U. & 1.620 \\
\hline Lipmes, S.A. & 1.500 \\
\hline RaiolaFuture, S.L. & 1.500 \\
\hline Solar Fotovoltaica Navarra, S.L. & 1.146 \\
\hline Jañez y Carrera, S.L. & 1.000 \\
\hline Isabel Sevillano Martín & 500 \\
\hline SolmayorEnergias Renovables, S.L. & 420 \\
\hline Meteo For Energy, S.L. & 300 \\
\hline Biertec 2000, S.L. & 100 \\
\hline Solarpack Corporación Tecnológica, S.L. & 100 \\
\hline Total & 3.909 .103 \\
\hline
\end{tabular}

Fuente: Boletín Oficial del Estado, nº 179, 28 de julio de 2017. 
Junto con las empresas adjudicatarias de las subastas, se están desarrollando otros grandes proyectos para la producción de electricidad de origen fotovoltaico. Reseñable es el caso de Extremadura, cuya Junta prevé durante los años 2018 y 2019 la construcción de 21 plantas fotovoltaicas que suman una potencia total de $1.800 \mathrm{MW}$, con una inversión de 1.200 millones de euros ${ }^{27}$.Esta región, además de con abundante radiación solar, cuenta con grandes propiedades rústicas con reducido aprovechamiento agrario que favorece su accesibilidad para el alquiler de las tierras durante tres décadas, así como una buena red eléctrica debido a la localización en su territorio de la central nuclear de Almaraz y de las centrales hidroeléctricas en las cuencas de los ríos Tajo y Guadiana (Mateos, B. y Leco, F., 2011).

La publicación en el Boletín Oficial del Estado de las Evaluaciones de Impacto Ambiental de estos proyectos de plantas fotovoltaicas permite conocer la magnitud de los mismos. Para ver algunos ejemplos se ha elaborado la tabla 16.

Tabla 16. Ejemplos de grandes plantas de energía solar fotovoltaica en desarrollo. 31-Julio-2019.

\begin{tabular}{|c|c|c|c|c|}
\hline Sociedad Promotora & $\begin{array}{l}\text { Denominación de la } \\
\text { instalación }\end{array}$ & $\begin{array}{l}\text { Localización. Munici- } \\
\text { pio y provincia }\end{array}$ & $\begin{array}{l}\text { Potencia } \\
\text { MW }\end{array}$ & $\begin{array}{l}\text { Presupueso. } \\
\text { Euros }\end{array}$ \\
\hline $\begin{array}{l}\text { Proyecto Solar Fran- } \\
\text { cisco Pizarro S.L. }\end{array}$ & Francisco Pizarro & $\begin{array}{l}\text { Torrecillas de la Tiesa } \\
\text { y Aldeacentenera } \\
\text { (Cáceres) }\end{array}$ & 590 & 506.496 .185 \\
\hline $\begin{array}{l}\text { Proyecto Núñez de } \\
\text { Balboa S.L. }\end{array}$ & Núñez de Balboa & $\begin{array}{l}\text { Usagre, Hinojosa del } \\
\text { Valle y Bienvenida } \\
\text { (Badajoz) }\end{array}$ & 500 & 189.394.216 \\
\hline $\begin{array}{l}\text { Promosolar Juwi } 17 \\
\text { S.L.U. }\end{array}$ & Mula & Mula (Murcia) & 494 & s.d. \\
\hline Dehesa Solar Sur S.L. & Oriol & $\begin{array}{l}\text { Ceclavín y Alcántara } \\
\text { (Cáceres) }\end{array}$ & 328 & 193.627 .362 \\
\hline $\begin{array}{l}\text { Genia Extremadura } \\
\text { Solar S.L. }\end{array}$ & Talayuela & $\begin{array}{l}\text { Talayuela, Rosalejo } \\
\text { y Navalmoral de la } \\
\text { Mata (Cáceres) }\end{array}$ & 300 & 180.677 .311 \\
\hline Valdesolar Hive S.L.* & Valdecaballeros & $\begin{array}{l}\text { Valdecaballeros (Ba- } \\
\text { dajoz) }\end{array}$ & 264 & 164.746 .606 \\
\hline $\begin{array}{l}\text { Novasol Invest La Isla } \\
\text { S.L. }\end{array}$ & La Isla & $\begin{array}{l}\text { Alcalá de Guadaira } \\
\text { (Sevilla) }\end{array}$ & 182 & s.d. \\
\hline $\begin{array}{l}\text { Iberia Termosolar } 1 \\
\text { S.L. }\end{array}$ & Bienvenida & Bienvenida (Badajoz) & 180 & 28.449 .567 \\
\hline Parque Solar S.L. & Arenales & $\begin{array}{l}\text { Cáceres, Casar de } \\
\text { Cáceres y Arroyo de } \\
\text { la Luz (Cáceres) }\end{array}$ & 121 & 98.272 .305 \\
\hline
\end{tabular}

Fuente: Boletín Oficial del Estado.

* En septiembre de 2018, Repsol anuncia que se ha hecho con la totalidad de ValdesolarHive, dentro de su estrategia de diversificación y ampliación de su presencia en el mercado eléctrico ${ }^{28}$. El 2 de noviembre de 2018 informa que ha incorporado los negocios no regulados de generación de electricidad de bajas emisiones (ciclos combinados y centrales hidroeléctricas) de Viesgo y su comercializadora para convertirse en un actor relevante del mercado eléctrico español ${ }^{29}$.

27. https://www.europapress.es/extremadura/noticia-junta-preve-construccion-21-plantas-fotovoltaicas-1200-millones-inversion-extremadura-antes-2020-20180719124818.html.

28. https://elperiodicodelaenergia.com/repsol-entra-en-el-negocio-solar-por-la-puerta-grande-adquiere-un-proyecto-fotovoltaico-de-264-mw-en-valdecaballeros/.

29. https://www.repsol.com/es/sala-prensa/notas-prensa/repsol-completa-compra-activos-viesgo-comercializadora.cshtml. 


\subsection{Líneas de apoyo y bonificaciones}

Los fondos públicos destinados a las ayudas a las energías renovables, casi siempre son de carácter regional, por lo que son los gobiernos autonómicos los que desarrollan los programas de incentivos. En líneas generales, la mayoría de las ayudas de las Comunidades Autónomas se apoyan en financiación del Fondo Europeo de Desarrollo Regional (FEDER). Un resumen de las principales ayudas ofrecidas por las distintas Comunidades Autónomas está disponible en el Anuario Fotovoltaico 2019 de la Asociación Nacional de Productores de Energía Fotovoltaica ${ }^{30}$. En los Boletines Oficiales de las distintas Administraciones se publican las normas y cuantías a conceder.

También los medios de comunicación aportan información sobre el apoyo de las Administraciones a la energía fotovoltaica a distintas escalas. Por ejemplo, el Portal de Eficiencia y Servicios Energéticos “Eseficiencia"(https://www.eseficiencia.es) ofrece todos los días noticias sobre ayudas y financiación de proyectos con energías renovables.

\section{LA EVOLUCIÓN DEL PRECIO DE LOS COMPONENTES DE UN SISTEMA FOTOVOLTAICO}

La tecnología solar fotovoltaica está en constante evolución y mejora técnica, siendo en la actualidad uno de los más relevantes polos de investigación a nivel global. La evolución y mejora técnica de los sistemas solares fotovoltaicos se centra sobre todo en los paneles solares fotovoltaicos, en las estructuras de fijación y orientación de dichos paneles, y en los equipos inversores solares/centrales de potencia, que son los elementos que más se han desarrollado (Asociación Nacional de Productores de Energía Fotovoltaica, 2019)

De la enorme caída del precio de los paneles fotovoltaicos dan buena cuenta los medios de información. El 7 de junio de 2018, el portal Energías Renovables difunde la noticia "Bloomberg pronostica para 2018 una caída acumulada de alrededor del 30\% en el precio de los paneles solares"31. Entre los años 2010 y 2017, el precio de los módulos solares cayó alrededor de un 80\%. Lo hizo de la mano de la I+D, que ha reducido extraordinariamente los costes de fabricación y distribución a la par que incrementaba la calidad del producto y su penetración en el mercado. El asesor de Bloomberg David Fickling califica de "dramática" la bajada de los precios en lo que va de año. Fickling señala tres ejemplos: $-43 \%$ en el caso de Jinko Solar, - $20 \%$ en producto de Canadian Solar y $-4,4 \%$ en el caso de Hanwha Q Cells, que son tres de los mayores fabricantes de paneles solares del mundo (Tabla 17). El precio del panel fotovoltaico ha caído más del $90 \%$ desde el año 2007, y el de los inversores fotovoltaicos ${ }^{32}$ a la mitad (Tabla 18).

Otro paso importante a la hora de reducir costes e impacto ambiental sobre el suelo donde se implantan las instalaciones, viene dado por la implantación de la técnica de hincado y la técnica de atornillado de la estructura metálica en el suelo. La introducción de este novedoso método para la construcción de grandes plantas fotovoltaicas ha supuesto un ahorro en los tiempos de ejecución muy alto, así como una importante reducción de costes, no solo derivados del diseño de la propia estructura, sino también provocados por la eliminación, entre otros, de los trabajos de vertido y colocación de zapatas de hormigón, que anteriormente servían de base para la sujeción de las estructuras de fijación y orientación de los módulos solares fotovoltaicos (Asociación Nacional de Productores de Energía Fotovoltaica, 2019).

\section{DISCUSIÓN Y CONCLUSIONES}

Según Villaring (2013), Giralt, Gago, Ordoñez y Muneer (2016) y Prados Velasco (2010), el sector de las energías renovables incide de forma importante en el crecimiento económico de muchas zonas rurales

30. https://anpier.org/wp-content/uploads/2019/06/anuario245x173-DEF.junio-2019-WEB.pdf.

31. https://www.energias-renovables.com/fotovoltaica/bloomberg-pronostica-para-2018-una-caida-acumulada-20180607.

32. Los equipos inversores transforman la energía captada por los paneles solares (corriente continua), en energía aprovechable para el consumo eléctrico (corriente alterna). 
españolas cuya renta per cápita es inferior a las áreas urbanas. Sin embargo, los resultados de este trabajo han permitido comprobar que la evolución de la potencia instalada para la producción de electricidad con energía solar fotovoltaica ha estado condicionada por la política energética del Gobierno de España, no siempre favorable a este tipo de energías, ni tampoco a los ámbitos rurales.

Tabla 17. Principales fabricantes de módulos fotovoltaicos en el mundo. 2018.

\begin{tabular}{|l|l|c|}
\hline \multicolumn{1}{|c|}{ Empresa } & \multicolumn{1}{|c|}{ País } & Entregas (MW) \\
\hline Jinko Solar & China & 11.380 \\
\hline JA Solar & China & 8.800 \\
\hline Trina Solar & China & 8.100 \\
\hline LONGi Solar & China & 7.200 \\
\hline Canadian Solar & China & 6.600 \\
\hline Hanwha Q-Cells & China & 5.600 \\
\hline RisenEnergy & China & 4.800 \\
\hline GCL-SI & China & 4.100 \\
\hline Telesun & China & 2.900 \\
\hline First Solar & Estados Unidos & 2.706 \\
\hline
\end{tabular}

Fuente: EurObserv’ER. BaromètrePhotovoltaïque 2019.

Tabla 18. Evolución del precio del panel solar y del inversor solar. Euros/vatio-pico. 2007-2019

\begin{tabular}{|l|c|c|}
\hline Año & Panel solar & Inversor solar \\
\hline 2007 & 3,30 & 0,130 \\
\hline 2008 & 2,50 & 0,128 \\
\hline 2009 & 1,68 & 0,125 \\
\hline 2010 & 1,40 & 0,117 \\
\hline 2011 & 1,10 & 0,110 \\
\hline 2012 & 0,75 & 0,107 \\
\hline 2013 & 0,60 & 0,090 \\
\hline 2014 & 0,45 & 0,085 \\
\hline 2015 & 0,42 & 0,079 \\
\hline 2016 & 0,38 & 0,070 \\
\hline 2017 & 0,35 & 0,064 \\
\hline 2018 & 0,34 & 0,057 \\
\hline 2019 & 0,28 & 0,055 \\
\hline
\end{tabular}

Fuente: Asociación Nacional de Productores de Energía Fotovoltaica (ANPIER). Anuario Fotovoltaico 2019. 
La localización de las explotaciones fotovoltaicas se ha producido preferentemente en la España peninsular, y dentro de ésta en su zona meridional, lo que se explica por la presencia de una mayor radiación solar y la estructura de la propiedad de la tierra que ha permitido disponer de explotaciones alquilables durante tres décadas (Diaz, M.P. et al., 2011; Fundación de Estudios sobre la Energía, 2010; Giralt et al., 2016).

Así, los $4.760 \mathrm{MW}$ instalados a finales de 2018 junto con los $3.900 \mathrm{MW}$ adjudicados en la subasta de julio de 2017 ( estarán en funcionamiento a finales de 2020) van a permitir alcanzar sobradamente el objetivo establecido en el Plan de Energías Renovables 2011-2020 para esta tecnología. Igualmente, la nueva producción eléctrica de origen solar fotovoltaica va a contibuir a que suba la aportación de las energías renovables al consumo de energía primaria en España, y con ello a la reducción de la producción en otras centrales térmicas de carbón o de ciclo combinado. Lo que supone una garantía para el abastecimiento en el momento en el que las centrales nucleares vayan finalizando su ciclo de vida productiva. En consecuencia, el incremento de la producción electrica de origen solar fotovoltaico dependerá no sólo de que se cumpla el Plan de energías Renovables 2011-2020 (Instituto para la Diversificación y Ahorro de Energía, 2011), sino sobre todo, de que las políticas del Gobierno de España sigan siendo favorables a la expansión de las energías renovables.

En conclusión, España, que se convirtió en un referente mundial en la producción de electricidad con energía solar fotovoltaica a mediados de la primera década de este siglo, vuelve a apostar por esta tecnología tanto para la generación en plantas de gran tamaño como para el autoconsumo. Si bien este significativo desarrollo de la energía fotovoltaica ha llevado a algunos autores a calificar dicho fenómeno "de burbuja" (Sevilla Jiménez, Golf Laville, y Driha, 2013; Fundación de estudios sobre la Energía, 2010; Mir, 2012; Gómez, Dopazo, y Fueyo, 2016) a causa de las políticas implantadas .

En realidad, el factor fundamental para el crecimiento tan destacado de la energía solar fotovoltaica en el Planeta ha sido la reducción de los precios de los componentes básicos de una instalación. Hoy en día un panel solar fotovoltaico cuesta una décima parte de lo que valía en el año 2007, y algo parecido ha sucedido con los inversores fotovoltaicos, que han reducido a la mitad su precio durante ese periodo; es decir, se han producido importantes innovaciones de proceso que han disminuido los tiempos de ejecución y los costes (Asociación nacional de productores de energía Fotovoltaica, 2019). Por ello, la energía solar fotovoltaica se ha vuelto muy competitiva en pocos años.

En la actualidad su nuevo empuje en España viene dado desde dos ámbitos. El tradicional, con la construcción de grandes plantas, tanto las acogidas al régimen de subastas como las que van por libre. Y por otra parte, el desarrollo de instalaciones de autoconsumo, una vez que el Gobierno de España ha creado un marco legal que favorece de verdad su implantación.

Las nuevas producciones de electricidad concedidas a través de las subastas, y que afectan tanto a centrales fotovoltaicas, como eólicas o de biomasa, en unos pocos años van a poner en el mercado eléctrico peninsular una cantidad de electricidad muy superior al incremento del consumo anual. En este sentido, los resultados de esta investigación indican que estos hechos pueden generar tensiones por la competencia con la producción de otras centrales, sobre todo las térmicas de carbón y ciclo combinado, y que se podrían resolver si se ampliaran las conexiones con Francia para poder exportar mayor volumen de electricidad a la Unión Europea.

De la misma manera que la conexión con Marruecos ha servido para la exportación de electricidad durante muchos años, aunque en la actualidad se utiliza para la importación de este recurso procedente de una central térmica de carbón recientemente inaugurada, que tiene una potencia de 1.400 MW, y que no está obligada a pagar derechos de emisión de dióxido de carbono.

\section{REFERENCIAS}

Asociación de Empresas de Energías Renovables (2018). Estudio del Impacto Macroeconómico de las Energías Renovables en España. 2017. Madrid.

Asociación Nacional de Productores de Energía Fotovoltaica (2019). Anuario Fotovoltaico 2019. Madrid.

Arán Carrión, J., Espín Estrella, A., Aznar Dols, F. y Ramos Ridao, A. (2008). The electricity production capacity of photovoltaic power plants and the selection of solar energy sites in Andalusia (Spain). Renewable Energy, vol. 33(4), 545-552. doi: https://doi.org/10.1016/j.renene.2007.05.041 
Baraja Rodríguez, E. y Herrero Luque, D. (2010). Energías renovables y paisaje en Castilla y León: estudio de caso. Nimbus. Revista de Climatología, Meteorología y Paisaje, 25-26, 21-42.

Baraja Rodríguez, E. y Herrero Luque, D. (2016). Energía, agua y territorio: de "cultivar energía solar" a "producir con energía solar" en los regadíos del Duero". En XV Coloquio Ibérico de Geografía (pp. 27-36). Murcia: Asociación de Geógrafos Españoles.

Castro-Gil Amigo, J., Tamames, R., Parejo Alfonso, L., Jiménez Piernas, C., Pascual Vives, F.J., Barnés Vázquez, J., Pérez de Ayala, L., Martín Pallín, J.A., García Breva, J. y Sánchez Corzo, J. (2014). Riesgo regulatorio en las energías renovables. Cizur Menor (Navarra): Thomson Reuters-Aranzadi.

Comisión Nacional de Energía (2008). Informe 30/2008 de la CNE en relación a la propuesta de real decreto de retribución de la actividad de producción de energía eléctrica mediante tecnología solar fotovoltaica para instalaciones posteriores a la fecha límite de mantenimiento de la retribución del Real Decreto 661/2007, de 25 de mayo, para dicha tecnología. Madrid, 29 de julio de 2008.

Costa Campi, M.T. (2016). Evolución del sector eléctrico español (1975-2015). Información Comercial Española, 889-890, 139-156

Dela Hoz. J., Boix, O., Martín, H., Martins, B. y Graells, M. (2010). Promotion of grid-connected photovoltaic systems in Spain: Performance analysis of the period 1998-2008. Renewable and Sustainable Energy Reviews, 14 (9), $2.547-2.563$. doi: https://doi.org/10.1016/j.rser.2010.07.059

Delgado, J. (2013). Las energías renovables: por qué sí y por qué no. Papeles de Economía Española, 134, 60-72.

Díaz Cuevas, M.P., Pita López, M.F. y Zoido Naranjo, F. (2011). El papel de Red Eléctrica en la definición de las potencialidades territoriales para la implantación de la energía eólica en Andalucía. En Gozálvez, V. y Marco, J.A. (Eds.) Energía y Territorio. Dinámicas y procesos. Comunicaciones. XXII Congreso de Geógrafos Españoles (pp. 109-118). Alicante: Asociación de Geógrafos Españoles-Colegio de Geógrafos-Universidad de Alicante.

Díaz Cuevas, M.P., Pita López, M.F., Fernández Tabales, A. y Limones Rodríguez, N. (2017). Energía eólica y territorio en Andalucía: diseño y aplicación de un modelo de potencialidad para la implantación de parques eólicos. Investigaciones Geográficas, 67, 9-29. doi: https://dx.doi.org/10.14198/INGEO2017.67.01

Díaz Mendoza, A.C., Larrea Basterra, M., Álvarez Pelegry, E. y Mosácula Atienza, C. (2015). De la liberalización (Ley 54/1997) a la reforma (Ley 24/2013) del sector eléctrico español. Bilbao: Cátedra de Energía de Orkestra -Instituto Vasco de Competitividad-Fundación Deusto, Documentos de Energía, Cuadernos Orkestra 2015-10.

Donoso Alonso, J. (2014). La energía fotovoltaica y la Reforma Eléctrica. Cuadernos de Energía, 42, 45-63.

Espejo Marín, C. (2004). La energía solar fotovoltaica en España. Nimbus. Revista de Climatología, Meteorología y Paisaje, 13-14, 5-32.

Espejo Marín, C. (2012). Energía y territorio: dinámicas y procesos. En Gozálvez Pérez, V. y Marco Molina, J.A. (Eds.) Geografía retos ambientales y territoriales. XXII. Congreso de Geógrafos Españoles (pp. 69-109). Alicante: Asociación de Geógrafos Españoles, Colegio de Geógrafos de España y Universidad de Alicante.

Espejo Marín, C. y García Marín, R. (2010). La energía solar termoeléctrica en España. Anales de Geografía de la Universidad Complutense, 30 (2), 81-105.

Espejo Marín, C. y García Marín, R. (2010). Agua y energía: producción hidroeléctrica en España. Investigaciones Geográficas, 51,107-129. doi: https://dx.doi.org/10.14198/INGEO2010.51.05

Espejo Marín, C. y García Marín, R. (2012). La energía eólica en la producción de electricidad en España. Revista de Geografía Norte Grande, 51, 115-136. doi: https://dx.doi.org/10.4067/S0718-34022012000100007

EurObserv'ER (2019). Baromètre Photovoltaïque 2019.

Fernández Cuesta, N. (2016). Veinte años de liberalización energética. Cuadernos de Energía, 50, 46-51.

Frolova, M., Espejo, C., Baraja, E. y Prados, M.J. (2014). Paisajes emergentes de las energías renovables en España. Boletín de la Asociación de Geógrafos Españoles, 66, 223-252.

Fundación de Estudios sobre Energía (2010).Energías renovables para la generación de electricidad en España. Madrid.

Galdós Urrutia, R. y Madrid Ruiz, F.J. (2009). La energía eólica en España y su contribución al desarrollo rural. Investigaciones Geográficas, 50, 93-108.doi: https://dx.doi.org/10.14198/INGEO2009.50.05

García Breva, J. (2019): La solar fotovoltaica domina el Planeta. En Asociación Nacional de Productores de Energías Renovables: Anuario Fotovoltaico 2019 (pp 22-23). Madrid. 
García Breva, J. y Martín Pallín, J.A. (2014). Seguridad Jurídica en Renovables. Fundamentos para una estrategia procesal ante la reforma eléctrica. Madrid: Asociación Nacional de Productores de Energía Fotovoltaica.

Girard, A., Gago, E.J., Ordoñez, J. y Muneer, T. (2016). Spain's energy outlook: A review of PV potencial and energy export. Renewable Energy, 86, 703-715. doi: 10.1016/j.renene.2015.08.074

Gómez, A., Dopazo, C. y Fueyo, N. (2016). El "coste de no hacer" planificación energética. El alto precio de la improvisación para el sector español de generación eléctrica y para los ciudadanos. Cuadernos de Energía, 50, 82-91.

González Hereza, N. (2015). La energía en Andalucía. Cuadernos de Energía, 46, 138-147.

Hernández Gonzálvez, C. y Hernández Luna, c. (2019). El 9\% de la electricidad será fotovoltaica en 2021. Energías Renovables, 178, 28-30.

Instituto para la Diversificación y Ahorro de la Energía (1999). Plan de Fomento de la Energías Renovables en España. Madrid: Ministerio de Ciencia y Tecnología

Instituto para la Diversificación y Ahorro de la Energía (2005). Plan de Energías Renovables en España 2005-2010. Madrid, Ministerio de Industria, Comercio y Turismo.

Instituto para la Diversificación y Ahorro de la Energía (2011). Plan de Energías Renovables2011-2020. Madrid: Ministerio de Industria, Comercio y Turismo.

Mateos Rodríguez, B. y Leco Berrocal, F. (2011). La producción de energía hidroeléctrica en Extremadura. En Gozálvez Pérez, V. y Marco Molina, J.A. (Eds.) Geografía retos ambientales y territoriales. XXII. Congreso de Geógrafos Españoles (pp. 215-226). Alicante: Asociación de Geógrafos Españoles, Colegio de Geógrafos de España y Universidad de Alicante.

Mérida Rodríguez, M., Reyes Corredera, S., Pardo García, S. y Zayas Fernández, B. (2015). Solar Photovoltaic Power in Spain. En Frolova, M., Prados, M.J. y Nadaï, A. (Eds.) Renewable Energies and European Landscapes. Lesson from Southerm European Cases (pp. 63-80). Berlín: Springer Science and Business Media. doi: https://doi.org/10.1007/97894-017-9843-3_4

Mérida Rodríguez, M., Lobón Martín, R. y Perles Roselló, M.J. (2015). The Production of Solar Photovoltaic Power and Its Landscape Dimension. The Case of Andalusia (Spain). En Frolova, M., Prados, M.J. y Nadaï, A. (Eds.) Renewable Energies and European Landscapes. Lesson from Southerm European Cases (pp. 255-257). Berlín: Springer Science and Business Media. doi: https://doi.org/10.1007/978-94-017-9843-3_14

Mir, P. (2012). Economía de la generación eléctrica solar. La regulación fotovoltaica y solar termoeléctrica en España. Cizur Menor (Navarra): Civitas. doi: https://doi.org/10.32796/cice.2012.83.6039

Prados Velasco, M.J. (2010). ¿Energías renovables o agricultura? Un análisis de la percepción ciudadana sobre los huertos y latifundios solares en Andalucía. Nimbus. Revista de Climatología, Meteorología y Paisaje, 25-26, 205-229.

Prados, M.J., Baraja, E., Frolova, M. y Espejo, C. (2012). Integración paisajística y territorial de las energías renovables. Ciudad y Territorio. Estudios Territoriales, 171, 127-143.

Red Eléctrica de España (2011). Rómulo. Interconexión eléctrica Península Baleares. Madrid.

Red Eléctrica de España (2019). Comprometidos con la energía inteligente El Sistema Eléctrico Español. 2018. Madrid.

Red Eléctrica de España (2019). Comprometidos con la energía inteligente. Las energías renovables en el Sistema Eléctrico Español. 2018. Madrid.

Salkind, N.J. y Rainwater, T. (2003). Exploring research. Upper Saddle River. New Jersey: Prentice Hall.

Sevilla Jiménez, M., Golf Laville, E. y Driha, O.M. (2013). Las energías renovables en España. Estudios de Economía Aplicada, 31 (1), 35-58.

Solar Power Europe (2018). Global Market Outlook. For Solar Power/2018-2022. Brussels.

Unión Española Fotovoltaica(2014). La energía fotovoltaica conquista el mercado. Informe Anual 2014. Madrid.

Unión Española Fotovoltaica (2015). La energía fotovoltaica una alternativa real. Informe Anual 2015. Madrid.

Unión Española Fotovoltaica. (2016). El tiempo de la energía solar fotovoltaica. Informe Anual 2016. Madrid.

Unión Española Fotovoltaica. (2017). El auge mundial de la fotovoltaica. Informe Anual 2017. Madrid.

Unión Fotovoltaica Española (2018). 2017: El inicio de una nueva era para el sector fotovoltaico. Madrid.

Villarig Tomás, J.M. (2013). Renovables o cómo renunciar al liderazgo. Cuadernos de Energía, 41, 119-123. 